\title{
Spatiotemporal response of crystals in x-ray Bragg diffraction
}

\author{
Yuri Shvyd'ko* and Ryan Lindberg ${ }^{\dagger}$ \\ Advanced Photon Source, Argonne National Laboratory, Argonne, Illinois 60439, USA
}

(Received 17 July 2012; published 4 October 2012)

\begin{abstract}
The spatiotemporal response of crystals in x-ray Bragg diffraction resulting from excitation by an ultrashort, laterally confined x-ray pulse is studied theoretically. The theory presents an extension of the analysis in symmetric reflection geometry [R. R. Lindberg and Y. V. Shvyd'ko, Phys. Rev. ST Accel. Beams 15, 050706 (2012)] to the generic case, which includes Bragg diffraction both in reflection (Bragg) and transmission (Laue) asymmetric scattering geometries. The spatiotemporal response is presented as a product of a crystal-intrinsic plane-wave spatiotemporal response function and an envelope function defined by the crystal-independent transverse profile of the incident beam and the scattering geometry. The diffracted wave fields exhibit amplitude modulation perpendicular to the propagation direction due to both angular dispersion and the dispersion due to Bragg's law. The characteristic measure of the spatiotemporal response is expressed in terms of a few parameters: the extinction length, crystal thickness, Bragg angle, asymmetry angle, and the speed of light. Applications to self-seeding of hard x-ray freeelectron lasers are discussed, with particular emphasis on the relative advantages of using either the Bragg or Laue scattering geometries. Intensity front inclination in asymmetric diffraction can be used to make snapshots of ultrafast processes with femtosecond resolution.
\end{abstract}

DOI: 10.1103/PhysRevSTAB.15.100702

PACS numbers: 41.50.+h, 41.60.Cr, 61.05.cp, 42.55.Vc

\section{INTRODUCTION}

The spatiotemporal response from crystals in symmetric $\mathrm{x}$-ray Bragg diffraction in reflection (Bragg) geometry has been studied in our recent publication [1]. Here, we extend the analysis to the generic case of asymmetric Bragg diffraction, both in reflection (Bragg) and transmission (Laue) geometries. "Asymmetric" means that the Bragg reflecting atomic planes are not parallel to the crystal surface.

Understanding the time dependence of x-ray Bragg diffraction in crystals has attracted much attention since the 1990s, as the advent of ultrafast (femtosecond short) $\mathrm{x}$-ray pulses becomes a close reality. The temporal and spatial dependence of diffraction was first calculated using the time dependent Takagi-Taupin equations. In particular, an analytical solution for the Bragg reflected wave from an infinitely thick crystal was derived by Chukhovskii and Förster [2]. Numeric calculations of the time dependence of Bragg diffraction from a crystal heated by a laser pulse was performed in [3]. Calculations of the time dependence by Fourier transforming the known monochromatic planewave solutions from the classical dynamical theory [4-11] have been considered in several publications [12-19]. In particular, Shastri et al. $[12,13]$ performed numerical calculations, which have revealed signature features of time

\footnotetext{
*shvydko@aps.anl.gov
}

${ }^{\dagger}$ lindberg@aps.anl.gov

Published by the American Physical Society under the terms of the Creative Commons Attribution 3.0 License. Further distribution of this work must maintain attribution to the author(s) and the published article's title, journal citation, and DOI. dependences of Bragg diffraction from crystals both in the Bragg-case and in the Laue-case geometries. Graeff and Malgrange [15,16] obtained analytical solutions for the time dependence of Bragg diffraction in the Laue geometry, with the refraction effects at the crystal exit surface taken into account. Bushuev [20] used Fourier transformation of the plane-wave solutions both in the frequency and momentum space, with the second order corrections included to more accurately account for the refraction effects, to obtain solutions in time and space and analyzed specific cases using numeric calculations.

The present paper is focused on the development of the theory and on the analysis of the spatiotemporal response of crystals in Bragg diffraction to the excitation by an ultrashort in time and spatially confined $\mathrm{x}$-ray pulse in the general case of asymmetric reflection (Bragg) and transmission (Laue) scattering geometries. The primary goal of the present study is to understand the general phenomenology of the spatiotemporal response by uncovering the dominant underlying physics and identifying the key physical parameters that determine the characteristic time and space scales involved. For this purpose, we derive comprehensive solutions that can be written in the general case as a product of two independent envelope functions: the first is a spatiotemporal plane-wave response function that depends only on the crystal and scattering geometry, while the second is an envelope that is specific to the initial conditions of the incident field. We derive analytical solutions for the response functions under several representative conditions, which clearly identifies the key physical parameters and makes possible a relatively simple interpretation of the general solution. 
When an ultrashort x-ray pulse instantaneously excites a perfect crystal, the output field is delayed and spread in time and space. The underlying reason behind this phenomenon is that each frequency component excites a monochromatic eigenwave field in the crystal that propagates along its own direction with its associated group velocity. As a consequence, the time response is intrinsically connected to the lateral spatial distribution of $x$ rays leaving the crystal upon Bragg diffraction both in the reflection or forward directions.

The paper is organized as follows. Comprehensive solutions for the spatiotemporal dependences of Bragg diffraction in reflection (Bragg) and transmission (Laue) asymmetric geometries are derived in Sec. II. In particular, in Sec. II B the solutions are derived for incident ultrashort $\mathrm{x}$-ray pulses with an unbounded plane-wave front, and in Sec. IIC for incident ultrashort $\mathrm{x}$-ray pulses with a bounded wave front. The solution for the bounded wave front is a product of a crystal-intrinsic and geometryspecific plane-wave spatiotemporal response function and an envelope function defined by the crystal-independent transverse profile of the incident beam and the scattering geometry. The response functions in the asymmetric Bragg geometry are derived analytically in Sec. III in the approximation of a nonabsorbing and thick $d \gg \bar{\Lambda}_{H}^{(\mathrm{s})}$ crystal (the crystal thickness $d$ being much larger than the characteristic extinction length of Bragg diffraction $\bar{\Lambda}_{H}^{(\mathrm{s})}$ to be more precisely defined below). The response functions in the asymmetric Laue geometry are derived in analytical form in Sec. IV for a nonabsorbing crystal of arbitrary thickness. Applications of the theory for self-seeding of x-ray freeelectron lasers (XFELs) and for ultrafast time measurements are discussed in Sec. V.

\section{COMPREHENSIVE SOLUTIONS FOR SPATIOTEMPORAL CRYSTAL RESPONSE}

We study here the spatiotemporal dependence of Bragg diffraction of ultrashort, laterally bound x-ray pulses from a system of parallel atomic planes in a flat crystal plate. Generic solutions are derived in three consecutive steps. First, well-known solutions of the dynamical theory of $\mathrm{x}$-ray Bragg diffraction in crystals [4-11,18] for incident monochromatic plane waves are briefly summarized in Sec. II A. In Sec. II B, we derive solutions for an initially ultrashort incident pulse with boundless plane-wave front. Finally, solutions are obtained in Sec. II C for an ultrashort incident pulse with confined wave front.

\section{A. Monochromatic plane-wave solutions}

One of most fundamental results of the dynamical theory of x-ray diffraction in perfect crystals is the concept of monochromatic eigenwave fields in crystals introduced by Ewald almost 100 years ago [4]. A similar concept in the electron theory of solids was introduced later by Bloch in
1928, which are therefore generally known as Bloch waves. In the simplest case, a linearly polarized incident monochromatic plane wave $\mathcal{E}_{\mathrm{i}} \exp \left(\mathrm{i} \boldsymbol{K}_{0} \boldsymbol{r}-\mathrm{i} \omega_{0} t\right)$, with a frequency $\omega_{0}$, and wave vector $\boldsymbol{K}_{0}=\left(\omega_{0} / c\right) \hat{\boldsymbol{u}}_{0}$, propagating along the optical axis $\hat{\boldsymbol{u}}_{0}$, excites in the crystal a wave field

$$
\mathcal{D}(\boldsymbol{r}, t)=\mathcal{E}_{\mathrm{i}} \exp \left(-\mathrm{i} \omega_{0} t\right) \sum_{H} R_{0 H} \exp \left[\mathrm{i}\left(\boldsymbol{K}_{0}+\boldsymbol{H}\right) \boldsymbol{r}\right],
$$

which is a sum of plane-wave components with wave vectors $\boldsymbol{K}_{0}+\boldsymbol{H}$ [21]. In the general case this sum involves all the reciprocal crystal lattice vectors $\boldsymbol{H}$ of the crystal. For each $\boldsymbol{H}$ there is a set of parallel atomic planes in the crystal perpendicular to $\boldsymbol{H}$ with an interplanar distance $d_{H}=2 \pi / H$ which actually composes the grating on which $\mathrm{x}$ rays diffract.

In the following we will consider the so-called two-wave case, where only two plane-wave field components are taken into account: the wave associated with forward Bragg diffraction $\boldsymbol{H}=0$, and one Bragg diffraction component with nonzero $\boldsymbol{H}$, for which $\left|\boldsymbol{K}_{0}+\boldsymbol{H}\right| \simeq\left|\boldsymbol{K}_{0}\right| \equiv K_{0}$, and for which therefore the relative difference

$$
\alpha=\frac{\left(\boldsymbol{K}_{0}+\boldsymbol{H}\right)^{2}-K_{0}^{2}}{K_{0}^{2}}=\frac{2 \boldsymbol{K}_{0} \boldsymbol{H}+\boldsymbol{H}^{2}}{K_{0}^{2}},
$$

has a very small magnitude. In particular, if $\alpha=0$ we obtain Bragg's law $2 \boldsymbol{K}_{0} \boldsymbol{H}+\boldsymbol{H}^{2}=0$, which can be also written as

$$
2 K_{0} \sin \theta=H .
$$

Here, $\theta$ is the glancing angle of incidence to the atomic planes, which equals the angle between $\boldsymbol{K}_{0}$ and the atomic planes such that $\boldsymbol{K}_{0} \boldsymbol{H}=-K_{0} H \sin \theta$. The quantity $\alpha$ (2) is an important parameter of the theory known as the deviation parameter, since it represents the deviation from Bragg's law.

We take the atomic planes associated with the reciprocal crystal lattice vector $\boldsymbol{H}$ to be oriented at an arbitrary (asymmetry) angle $\eta$ with respect to the crystal surface, as shown schematically by white parallel lines in Figs. 1(a) and 1(b). Figure 1(a) represents diffraction in the reflection or Bragg scattering geometry, which is characterized by the diffracted wave field exiting the crystal on the same side as the incident wave, while the forward diffracted wave field propagates along the incident wave direction and exits the crystal on the opposite side. Figure 1(b) represents diffraction in the transmission or Laue scattering geometry, for which both the diffracted and forward diffracted wave fields exit the crystal from the surface opposite that of the incident wave. The crystal surfaces are defined by the unit normal vector $\hat{z}$ internal to the entrance surface. We restrict our theory to the case in which $\boldsymbol{K}_{0}$ is directed in the plane composed by $\boldsymbol{H}$ and $\hat{z}$, hereafter referred to as dispersion plane.

The dynamical theory of $\mathrm{x}$-ray diffraction allows one to calculate for each incident monochromatic plane-wave 

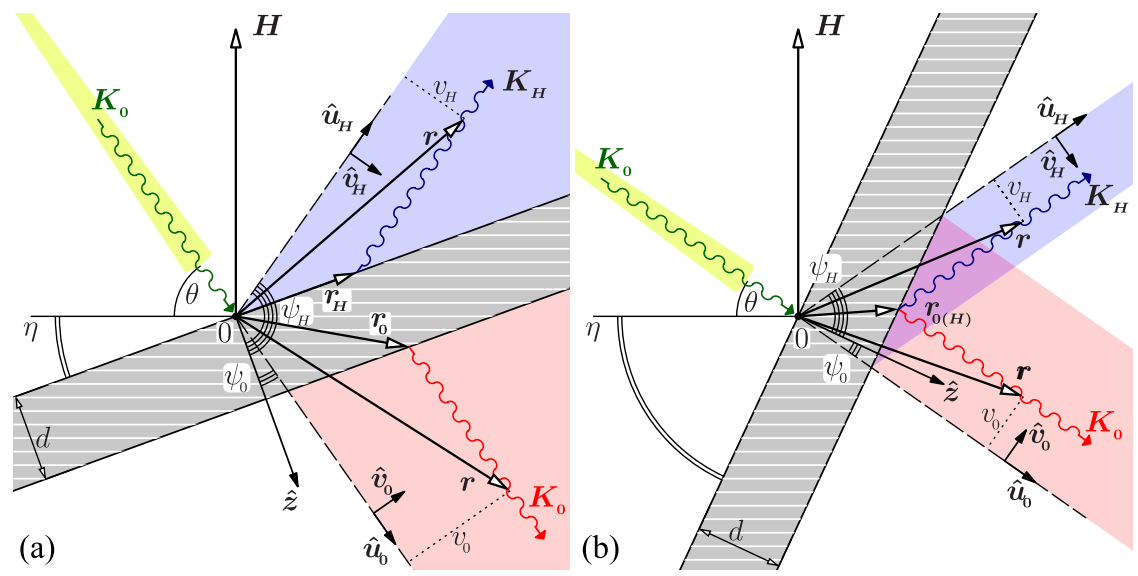

FIG. 1. Schematic presentation of two-beam x-ray Bragg diffraction from crystals (a) in the reflection (or Bragg) scattering geometry, and (b) in the transmission (or Laue) scattering geometry. The glancing angle of incidence to the reflecting atomic planes is $\theta$, and the angle between the reflecting planes and the crystal surface (the asymmetry angle) is $\eta$. The propagation direction $\hat{\boldsymbol{u}}_{H}$ of the Bragg reflected beam composes an angle $\psi_{H}$ with the internal normal $\hat{z}$ to the crystal surface. The angle $\psi_{0}$ between $\hat{z}$ and the direction $\hat{\boldsymbol{u}}_{0}$ of propagation of the incident beam is defined by the relationship $\psi_{0}+\psi_{H}=2 \theta$. For the scattering configurations shown in (a) and (b) $\psi_{H}=\pi / 2+\theta-\eta$, and therefore $\psi_{0}=\theta+\eta-\pi / 2$. The permitted range of the asymmetry angle $\eta$ in the Braggcase geometry is $-\theta<\eta<\theta$, while in the Laue-case geometry it is $\theta<\eta<\pi-\theta$. Negative $\eta$ in the Bragg-case geometry corresponds to a configuration complimentary to that shown in (a) with the incident and reflected beams reversed. Pink and light blue areas indicate regions where the forward diffracted and diffracted beams can propagate. See text for other details and definitions.

component $\exp \left\{-\mathrm{i}\left[\omega_{0} t-\boldsymbol{K}_{0} \boldsymbol{r}\right]\right\}$ both the monochromatic wave field of forward Bragg diffraction

$$
\mathcal{D}_{0}^{(\mathrm{m})}\left(\boldsymbol{r}_{0}, t\right)=\mathcal{E}_{\mathrm{i}} \mathrm{e}^{-\mathrm{i}\left[\omega_{0} t-\boldsymbol{K}_{0} \boldsymbol{r}_{0}\right]} R_{00}\left(\omega_{0}\right)
$$

at any point $\boldsymbol{r}_{0}$ on the rear surface of the crystal, and the monochromatic wave field of Bragg diffraction

$$
\mathcal{D}_{H}^{(\mathrm{m})}\left(\boldsymbol{r}_{H}, t\right)=\mathcal{E}_{\mathrm{i}} \mathrm{e}^{-\mathrm{i}\left[\omega_{0} t-\left(\boldsymbol{K}_{0}+\boldsymbol{H}\right) \boldsymbol{r}_{H}\right]} R_{0 H}\left(\omega_{0}\right) .
$$

The Bragg diffracted field (5) is determined at any point on the entrance surface $\boldsymbol{r}_{H}$ in the case of Bragg geometry shown in Fig. 1(a), or at any point on the rear surface $\boldsymbol{r}_{H}$ for the Laue geometry shown in Fig. 1(b). Here, $R_{0 \mathcal{H}}\left(\omega_{0}\right)$ are diffraction $(\mathcal{H}=H)$ or forward diffraction $(\mathcal{H}=0)$ crystal amplitudes which are functions not only of $\omega_{0}$ (whose dependence we explicitly indicate since it is most relevant to the discussion of the time behavior), but are also functions of the crystal thickness $d$, the direction and magnitude of $\boldsymbol{K}_{0}$, the asymmetry angle $\eta$, etc. At this point we are concerned with deriving general expressions for the spatiotemporal response of the crystal in x-ray Bragg diffraction, and so do not yet specify these reflection amplitudes; explicit expressions for the amplitudes $R_{0 \mathcal{H}}$ are presented in Secs. III and IV.

The in-crystal monochromatic wave field components $\mathcal{D}_{\mathcal{H}}^{(\mathrm{m})}\left(\boldsymbol{r}_{\mathcal{H}}, t\right)$ given by Eqs. (4) and (5) can also be used to calculate the field at any point $\boldsymbol{r}$ outside of the crystal. Using the continuity of the wave fields at the crystalvacuum interface determined by the extremities of the vectors $\boldsymbol{r}_{\mathcal{H}}$, the forward diffracted and diffracted wave fields in an arbitrary point $\boldsymbol{r}$ in vacuum can be written as

$$
\mathcal{E}_{\mathcal{H}}^{(\mathrm{m})}(\boldsymbol{r}, t)=\mathcal{D}_{\mathcal{H}}^{(\mathrm{m})}\left(\boldsymbol{r}_{\mathcal{H}}, t\right) \mathrm{e}^{\mathrm{i} \boldsymbol{K}_{\mathcal{H}}\left(\boldsymbol{r}-\boldsymbol{r}_{\mathcal{H}}\right)}
$$

where $\boldsymbol{K}_{\mathcal{H}}$ is the wave vector of the forward diffracted $(\mathcal{H}=0)$ or diffracted $(\mathcal{H}=H)$ field in vacuum. To match phase fronts, the components of the in-crystal wave vectors can differ from the vacuum wave vectors only by a component along the crystal normal $\hat{z}$. Since we assume that the crystal entrance and exit surfaces are parallel, this component is zero for the wave vector $\boldsymbol{K}_{0}$, and makes it equivalent to the vacuum wave vector $\boldsymbol{K}_{0}$ of the incident plane wave. However, this component is not zero for the vacuum wave vector of the diffracted wave. In the general case it can be written as

$$
\boldsymbol{K}_{H}=\boldsymbol{K}_{0}+\boldsymbol{H}+\Delta_{H} \hat{z} .
$$

The component $\Delta_{H} \hat{z}$ can be understood as an additional momentum transfer due to refraction at the crystal-vacuum interface, and Eq. (7) as momentum conservation in scattering from the crystal. Since Bragg diffraction is an elastic scattering process and the vacuum is homogeneous, the magnitude of the vacuum wave vector $\boldsymbol{K}_{H}$ of the diffracted wave should be equal to the vacuum wave vector of the incident plane wave: $\left|\boldsymbol{K}_{H}\right|=\left|\boldsymbol{K}_{0}\right| \equiv K_{0}$. From this condition and Eqs. (7) and (2), we find $\Delta_{H}=$ $K_{0}\left(-\tilde{\gamma}_{H} \pm \sqrt{\tilde{\gamma}_{H}^{2}-\alpha}\right)$ (see [18] for details), where $\tilde{\gamma}_{H}=$ $\hat{z}\left(\boldsymbol{K}_{0}+\boldsymbol{H}\right) / K_{0}$. For small $\alpha$, the additional momentum transfer can be closely approximated by a Taylor expansion in $\alpha$ : 


$$
\Delta_{H}=-K_{0} \frac{\alpha}{2 \tilde{\gamma}_{H}}-K_{0} \frac{\alpha^{2}}{8 \tilde{\gamma}_{H}^{3}}+\cdots .
$$

Now from Eqs. (4)-(7) we can write for the monochromatic forward diffracted and diffracted wave fields:

$$
\begin{gathered}
\mathcal{E}_{0}^{(\mathrm{m})}(\boldsymbol{r}, t)=\mathcal{E}_{\mathrm{i}} \mathrm{e}^{-\mathrm{i}\left[\omega_{0} t-\boldsymbol{K}_{0} \boldsymbol{r}\right]} R_{00}\left(\omega_{0}\right), \\
\mathcal{E}_{H}^{(\mathrm{m})}(\boldsymbol{r}, t)=\mathcal{E}_{\mathrm{i}} \mathrm{e}^{-\mathrm{i}\left[\omega_{0} t-\left(\boldsymbol{K}_{0}+\boldsymbol{H}\right) \boldsymbol{r}\right]} \mathrm{e}^{\mathrm{i} \Delta_{H}\left(\boldsymbol{r}-\boldsymbol{r}_{H}\right) \hat{z}} R_{0 H}\left(\omega_{0}\right) .
\end{gathered}
$$

Here $\left(\boldsymbol{r}-\boldsymbol{r}_{H}\right) \hat{z}$ is the shortest distance from the observation point $\boldsymbol{r}$ to the crystal surface. Since $\hat{z}$ is perpendicular to the crystal surface, it is actually independent of $\boldsymbol{r}_{H}$, and $\left(\boldsymbol{r}-\boldsymbol{r}_{H}\right) \hat{z}=\boldsymbol{r} \hat{z}$ if the extremity of $\boldsymbol{r}_{H}$ is on the entrance surface, and $\left(\boldsymbol{r}-\boldsymbol{r}_{H}\right) \hat{z}=\boldsymbol{r} \hat{z}-d$ if the extremity of $\boldsymbol{r}_{H}$ is on the rear surface. Here $d$ is the crystal thickness.

\section{B. Ultrashort incident pulse with boundless plane-wave front}

To study the spatiotemporal dependence of x-ray diffraction, in the next step we investigate the response to an initially ultrashort (instantaneous) x-ray pulse. We assume that the $\mathrm{x}$-ray pulse propagates along the direction of the unit vector $\hat{\boldsymbol{u}}_{0}$ which is in the dispersion plane built by vectors $\boldsymbol{H}$ and $\hat{z}$, and that the propagation direction $\hat{\boldsymbol{u}}_{0}$ makes a glancing angle of incidence $\theta$ with respect to the reflecting atomic planes.

The x-ray pulse is ultrashort, has a vector amplitude $\mathcal{E}_{\mathrm{i}}$, and has an infinite extent in the transverse direction $\hat{\boldsymbol{v}}_{0} \perp \hat{\boldsymbol{u}}_{0}$. In this case, the pulse at time $t$ and spatial point $\boldsymbol{r}$ can be presented by the delta function $\delta(\tau)$ of the argument $\tau=t-\hat{\boldsymbol{u}}_{0} \boldsymbol{r} / c$, with $c$ the speed of light in vacuum. The latter is equivalent to an infinite sum of monochromatic plane-wave components given by

$$
\begin{gathered}
\mathcal{E}_{\mathrm{i}} \mathrm{e}^{-\mathrm{i} \omega \tau} \delta(\tau)=\mathcal{E}_{\mathrm{i}} \int_{-\infty}^{\infty} \frac{d \Omega}{2 \pi} \mathrm{e}^{-\mathrm{i}(\omega+\Omega) \tau}, \\
\tau=t-\frac{\hat{\boldsymbol{u}}_{0} \boldsymbol{r}}{c}, \quad \omega+\Omega=\omega_{0}, \quad \boldsymbol{K}_{0}=\frac{\omega_{0}}{c} \hat{\boldsymbol{u}}_{0} .
\end{gathered}
$$

Here, we single out one plane-wave component with a frequency $\omega$ (we assume $\omega \gg \Omega$ ), which we define to satisfy the condition $\alpha=0$ defined in Eq. (2). In other words, we are selecting out the frequency for which Bragg's law (3) is fulfilled. For the frequency $\omega$ Bragg's law reads $\omega \sin \theta=H c / 2$. With this convention, the deviation parameter $\alpha$ (2) can be presented as

$$
\alpha=-4 \frac{\Omega}{\omega} \sin ^{2} \theta\left(1-2 \frac{\Omega}{\omega}+\cdots\right),
$$

and the additional momentum transfer $\Delta_{H}(8)$ as

$$
\frac{\Delta_{H}}{\omega / c}=\frac{2 \sin ^{2} \theta}{\gamma_{H}} \frac{\Omega}{\omega}\left[1-\frac{\Omega}{\omega}\left(b+\frac{\sin ^{2} \theta}{\gamma_{H}^{2}}\right)+\cdots\right],
$$

where

$$
\begin{array}{rlrl}
b & =\frac{\gamma_{0}}{\gamma_{H}}, & & \gamma_{0}=\hat{z} \hat{\boldsymbol{u}}_{0}, \\
\gamma_{H} & =\hat{z} \hat{\boldsymbol{u}}_{H}, & \hat{\boldsymbol{u}}_{H}=\hat{\boldsymbol{u}}_{0}+\frac{\boldsymbol{H}}{\omega / c}
\end{array}
$$

are the so-called asymmetry factor $b$, and direction cosines

$$
\gamma_{0} \equiv \cos \psi_{0}, \quad \gamma_{H} \equiv \cos \psi_{H} .
$$

In almost all of what follows we retain only the terms linear in the small quantity $\Omega / \omega$ for the expressions for $\alpha$ (13) and $\Delta_{H}$ (14), but we also present in Sec. II B 2 a brief description of the physics of the quadratic terms and how they can be included.

\section{Linear approximation}

Time $t=0$ is defined hereafter as the moment when the wave front hits the point $\boldsymbol{r}=0$ on the crystal. Similar to (11), the spatiotemporal response of the crystal in Bragg diffraction $\mathcal{E}_{\mathcal{H}}(\boldsymbol{r}, t)$, both for diffracted $(\mathcal{H}=H)$ and forward diffracted ( $\mathcal{H}=0$ ) components, can be calculated as an integral (in fact, a Fourier integral):

$$
\mathcal{E}_{\mathcal{H}}(\boldsymbol{r}, t)=\int_{-\infty}^{\infty} \frac{d \Omega}{2 \pi} \mathcal{E}_{\mathcal{H}}^{(\mathrm{m})}(\boldsymbol{r}, t)
$$

over the monochromatic components (9) and (10). A similar procedure was also applied in the previous publications [12-20,22]. Using (9) and (10), we obtain

$$
\begin{gathered}
\mathcal{E}_{\mathcal{H}}(\boldsymbol{r}, t)=\mathcal{E}_{\mathrm{i}} \mathrm{e}^{-\mathrm{i} \omega \tau_{\mathcal{H}} G_{0 \mathcal{H}}}\left(\xi_{\mathcal{H}}\right), \\
G_{0 \mathcal{H}}\left(\xi_{\mathcal{H}}\right)=\int_{-\infty}^{\infty} \frac{d \Omega}{2 \pi} \mathrm{e}^{-\mathrm{i} \Omega \xi_{\mathcal{H}}} R_{0 \mathcal{H}}(\omega+\Omega),
\end{gathered}
$$

where

$$
\begin{gathered}
\tau_{\mathcal{H}}=t-\frac{\hat{\boldsymbol{u}}_{\mathcal{H}} \boldsymbol{r}}{c} \\
\xi_{\mathcal{H}}=t-\frac{\hat{\boldsymbol{u}}_{0} \boldsymbol{r}}{c}-2 \sin ^{2} \theta \frac{\left(\boldsymbol{r}-\boldsymbol{r}_{H}\right) \hat{z}}{c \gamma_{H}} \delta_{\mathcal{H} H} .
\end{gathered}
$$

Here, $\delta_{\mathcal{H} H}$ is the Kronecker delta, which equals one only if $\mathcal{H}=H$, otherwise it is zero.

The plane-wave crystal response functions $G_{0 \mathcal{H}}\left(\xi_{\mathcal{H}}\right)$ in (18) represent the spatiotemporal dependence of Bragg diffraction $(\mathcal{H}=H)$ or forward Bragg diffraction ( $\mathcal{H}=0)$ to the excitation by a $\delta$-function-short incident radiation pulse with boundless transverse wave front.

Two spatiotemporal variables are introduced in (17)-(20). The variable $\xi_{\mathcal{H}}(\mathcal{H}=0, H)(20)$ is the argument of the response function (18). The spatiotemporal variable $\tau_{\mathcal{H}}(\mathcal{H}=0, H)(19)$ is in the argument of the exponential function of Eq. (17).

The spatiotemporal variable $\tau_{\mathcal{H}}(\mathcal{H}=0, H)$ represents the difference between the absolute time $t$ and the time $\hat{\boldsymbol{u}}_{\mathcal{H}} \boldsymbol{r} / c$ the plane-wave front, propagating along the optical axis $\hat{\boldsymbol{u}}_{\mathcal{H}}$ from $\boldsymbol{r}=0$, would need to reach an arbitrary point 


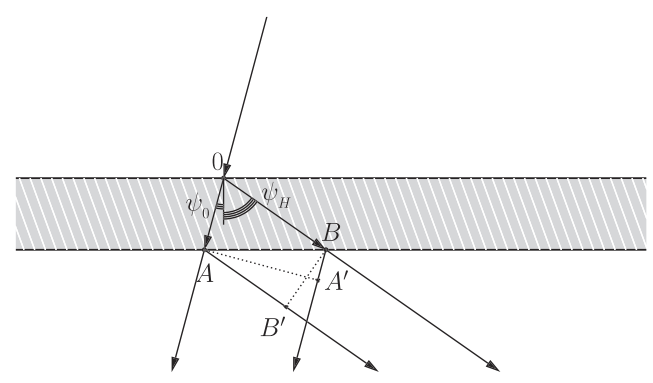

FIG. 2. Schematic presentation of plane-wave front paths (solid vector lines) in Laue-case Bragg diffraction and forward diffraction from a crystal plate. The total duration of forward diffraction $\left(0 B A^{\prime}-0 A\right) / c=\mathcal{T}_{d}$ (21), while total duration of diffraction is $\left(0 A B^{\prime}-0 B\right) / c=\mathcal{T}_{d} / b$. The lateral spread of forward Bragg diffraction is $A A^{\prime}=d \sin 2 \theta / \gamma_{H}$, and the lateral spread of Bragg diffraction is $B B^{\prime}=d \sin 2 \theta / \gamma_{0}$.

$\boldsymbol{r}$ outside of the crystal, assuming the propagation is in vacuum and the wave front is perpendicular to the optical axis $\hat{\boldsymbol{u}}_{\mathcal{H}}$. In other words, $\tau_{\mathcal{H}}$ is the time delay for the radiation field at point $\boldsymbol{r}$ we are interested in, as compared to the trivial propagation of the pulse in vacuum along $\hat{\boldsymbol{u}}_{\mathcal{H}}$.

The variable $\xi_{0}$ is equivalent to $\tau_{0}$, while the meaning of $\xi_{H}$ may not be immediately evident. To gain more insight, we rewrite $\xi_{H}$ in an equivalent form using (19), (20), and (15), and Bragg's law $\omega=H c /(2 \sin \theta)(3)$ :

$\xi_{\mathcal{H}}=\tau_{H}+2 \sin \theta\left(\frac{\boldsymbol{H}}{H}-\frac{\sin \theta}{\gamma_{H}} \hat{z}\right) \frac{\boldsymbol{r}}{c}+\mathcal{T}_{d} \delta_{B L}$

$\mathcal{T}_{d}=\frac{2 d \sin ^{2} \theta}{c\left|\gamma_{H}\right|}$

$\delta_{B L}= \begin{cases}0 & \text { in Bragg geometry } \\ 1 & \text { in Laue geometry. }\end{cases}$

The parameter $\mathcal{T}_{d}$ is a characteristic measure of time in Bragg diffraction associated with the crystal thickness $d$. In the Laue-case geometry, $\mathcal{T}_{d}$ is equal to the total duration of forward Bragg diffraction, which is given by the difference in path lengths for the wave to propagate along $0 B A^{\prime}$ and $0 A$, as shown schematically in Fig. 2. The total duration of Bragg diffraction in Laue-case geometry is determined by the difference in path lengths $0 A B^{\prime}$ and $O B$, which equals $\mathcal{T}_{d} / b$ as can be derived from schematic in Fig. 2. Although the last term in (21) vanishes in the Braggcase geometry, the parameter $\mathcal{T}_{d}$ continues to play an important role. Unlike the Laue-case geometry, diffraction in Bragg-case geometry is not limited in time, because multiple reflections from the front and rear surfaces take place $[7,10,11,23]$ as shown schematically in Fig. 3 . Accordingly, the parameter $\mathcal{T}_{d}$ is a characteristic measure of time associated with crystal thickness in the Bragg-case geometry, where it measures the time between multiple reflections as explained in the caption to Fig. 3.

Using Fig. 1, we can express $\hat{z}$ and $\boldsymbol{H} / H$ in (21) in terms of the unit vector $\hat{\boldsymbol{u}}_{H}$ along the diffraction optical axis,

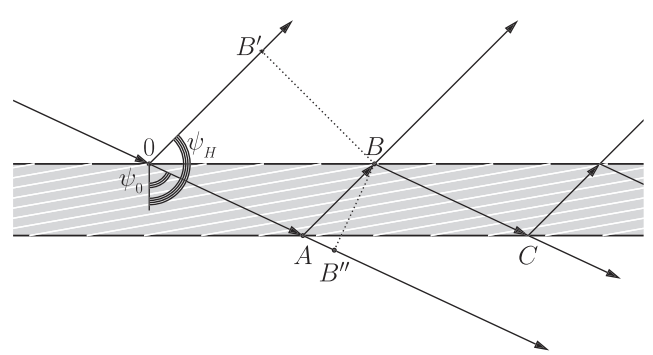

FIG. 3. Schematic presentation of plane-wave front paths (solid vector lines) with possible multiple internal reflections from the rear and front crystal surfaces in Bragg-case Bragg diffraction and forward Bragg diffraction from a crystal plate. The forward diffracted wave $0 A B C$ originating from the front surface reflection in $B$ is delayed by $\left(0 A B-0 B^{\prime \prime}\right) / c=\mathcal{T}_{d}(21)$, as compared to the primary forward diffracted wave $0 B^{\prime \prime}$. The diffracted wave $0 A B$ originating from the rear surface reflection in $A$ is delayed by $\left(0 A B-0 B^{\prime}\right) / c=\mathcal{T}_{d} /|b|$ compared to the primary diffracted wave $0 B^{\prime}$.

and the unit vector $\hat{\boldsymbol{v}}_{H}$ perpendicular to the axis as follows: $\hat{z}=\cos \psi_{H} \hat{\boldsymbol{u}}_{H}+\sin \psi_{H} \hat{\boldsymbol{v}}_{H}$, and $\boldsymbol{H} / H=\sin \theta \hat{\boldsymbol{u}}_{H}-\cos \theta \hat{\boldsymbol{v}}_{H}$. With these, the third term in (21) can be presented as

$$
\begin{aligned}
2 \sin \theta\left(\frac{\boldsymbol{H}}{H}-\frac{\sin \theta}{\gamma_{H}} \hat{z}\right) & =D \hat{\boldsymbol{v}}_{H}, \\
D=-\frac{2 \sin \theta \sin \eta}{\gamma_{H}} & \equiv-(1+b) \tan \theta .
\end{aligned}
$$

Equation (21) for $\xi_{H}$ thus can be now written as

$$
\xi_{H}=\tau_{H}+D \frac{\hat{\boldsymbol{v}}_{H} \boldsymbol{r}}{c}+\mathcal{T}_{d} \delta_{B L},
$$

where $\tau_{H}=t-\hat{\boldsymbol{u}}_{H} \boldsymbol{r} / c$ (19).

The quantity $D$ in (22) and (23) is the normalized angular dispersion rate. It is a measure of the variation of the propagation direction $K / K_{0} H$ of the diffracted wave (7) as a function of the incident photon energy $\hbar K_{0} / c$, assuming a fixed direction $\boldsymbol{K}_{0} / K_{0}$ of the incident wave vector. Indeed, using (7) we obtain

$$
\delta\left(\boldsymbol{K}_{H} / K_{0}\right)=-D \hat{\boldsymbol{v}}_{H}\left(\delta K_{0} / K_{0}\right),
$$

see [18] for details. We note that the normalized angular dispersion rate $D$ is zero only in Bragg-case symmetric scattering geometry with $\eta=0$. In all other cases, including the "symmetric" Laue geometry $\left(\eta=90^{\circ}\right)$, it is nonzero. Depending on the sign of $\eta, D$ can take positive or negative values in the Bragg-case geometry. In the Lauecase geometry $\eta>\theta$ and $D$ is therefore always negative.

From Eq. (23) we conclude that the spatiotemporal variable $\xi_{H}=\tau_{H}+D \hat{\boldsymbol{v}}_{H} \boldsymbol{r} / c+\mathcal{T}_{d}$ contains in addition to $\tau_{H}$ an important term $D \hat{\boldsymbol{v}}_{H} \boldsymbol{r} / c$ which describes a spatially lateral (perpendicular to the diffraction axis $\hat{\boldsymbol{u}}_{H}$ ) amplitude modulation $G_{0 H}\left(\xi_{H}\right)$ of the diffracted radiation field (18). The amplitude modulation occurs due to interference of different spectral components propagating in different directions, which arise from the angular dispersion due to the additional momentum transfer 
$\Delta_{H}$-Eqs. (7) and (14). The effect is very generic and vanishes only in one case, in symmetric Bragg geometry when $\eta=0$-Fig. 1(a). We will refer to this effect as the angular dispersive lateral spatial modulation of the diffracted wave field (here by angle we mean the angle of reflection rather than the angle of incidence).

\section{Nonlinear phase}

In our preceding discussion we have focused on the phase contributions that are linear in the frequency difference $\Omega$. While this is a very good approximation for the wave fields in vacuum close to the crystal surface, the nonlinear $\left(\sim \Omega^{2}\right)$ contributions inherent in the additional momentum transfer $\Delta_{H}$ give rise to additional physics over the potentially large propagation distances between the crystal surface and any experimental sample/detector. Here, we briefly quantify this effect and summarize its physical origin.

Using Eq. (15), the definition of $\psi_{0}$ and $\psi_{H}$ given in the caption of Fig. 1, along with the definition of $D(22)$, the expression for the additional momentum transfer (14) can be rewritten as

$$
\frac{\Delta_{H}}{\omega / c}=\frac{2 \sin ^{2} \theta}{\gamma_{H}} \frac{\Omega}{\omega}-\frac{D^{2}}{2 \gamma_{H}}\left(\frac{\Omega}{\omega}\right)^{2}+\cdots .
$$

Including the quadratic phase dependence in Eq. (24), the wave fields (17) and (18) can be presented as

$$
\begin{gathered}
\mathcal{E}_{\mathcal{H}}(\boldsymbol{r}, t)=\mathcal{E}_{\mathrm{i}} \mathrm{e}^{-\mathrm{i} \omega \tau_{\mathcal{H}}} X_{0 \mathcal{H}}\left(\xi_{\mathcal{H}}\right), \\
X_{0 \mathcal{H}}\left(\xi_{\mathcal{H}}\right)=\int_{\infty}^{\infty} \frac{\mathrm{d} \Omega}{2 \pi} \mathrm{e}^{-\mathrm{i} \Omega \xi_{\mathcal{H}}} R_{0 \mathcal{H}}(\Omega) S(\Omega), \\
S(\Omega)=\exp \left[-\frac{\mathrm{i} D^{2} \rho}{2 c \omega \gamma_{H}} \Omega^{2}\right],
\end{gathered}
$$

where $\rho=\left(\boldsymbol{r}-\boldsymbol{r}_{H}\right) \hat{z}$ is the shortest distance from the observation point to the crystal surface, so that $\rho / \gamma_{H}$ is the propagation distance along the optical axis. We note that the expression for the nonlinear in $\Omega$ phase factor $S(\Omega)$ in (27) is in agreement with that obtained earlier by Bushuev in [20], despite the different approaches used. With the help of the Fourier convolution theorem we obtain for $X_{0 \mathcal{H}}\left(\xi_{\mathcal{H}}\right)$ :

$$
\begin{gathered}
X_{0 \mathcal{H}}\left(\xi_{\mathcal{H}}\right)=\int_{0}^{\infty} d \xi G_{0 \mathcal{H}}(\xi) F\left(\xi_{\mathcal{H}}-\xi\right), \\
F\left(\xi_{\mathcal{H}}-\xi\right)=\sqrt{\frac{2 c \omega \gamma_{H}}{i D^{2} \rho}} \exp \left[\frac{i c \omega \gamma_{H}}{2 \rho} \frac{(\xi H-\xi)^{2}}{D^{2}}\right] .
\end{gathered}
$$

Here $G_{0 \mathcal{H}}$ is the crystal response given by Eq. (18), while $F(\xi)$ is the Fourier transform of $S(\Omega)$. The convolution is similar in form to that associated with paraxial evolution for the field $G_{0 \mathcal{H}}(\xi)$, with the Fourier transform of $S$ serving as the associated Green function, $\rho / \gamma_{H}$ the propagation distance along the optical axis, and $\xi / D$ playing the role of the "transverse" coordinate.

In fact, the factor $1 / D$ gives the amount the inclined intensity front will spread in time due to natural vacuum diffraction broadening along the transverse coordinate $\hat{\boldsymbol{u}}_{H}$. In symmetric Bragg (reflection) geometry the reflected intensity and phase fronts are parallel, $D=0$ and the time structure remains invariant, while in all other cases $D \neq 0$ and the separated, inclined intensity fronts will tend to smear together as the distance from the crystal $\rho$ increases. The maximum distance over which the linear approximation holds and we can ignore this spreading can be estimated as

$$
\rho \ll \frac{2 c \gamma_{H}}{D^{2} \omega}\left(\frac{\omega}{\Omega_{\mathrm{B}}}\right)^{2},
$$

where $\Omega_{\mathrm{B}}$ is the typical frequency range of Bragg diffraction, i.e., the range in $\Omega$ over which $R_{0 \mathcal{H}}(\Omega)$ is appreciable. For example, if $\Omega_{\mathrm{B}} / \omega \simeq 10^{-4}$, the radiation wavelength $\lambda=2 \pi \omega / c \simeq 1 \AA$, and $\gamma_{H} / D^{2} \approx 1$, the linear approximation breaks down at the rather small distance $\rho \approx 1 \mathrm{~cm}$ from the crystal. The smaller the bandwidth of the Bragg reflection $\Omega_{\mathrm{B}}$, the larger is the distance from the crystal over which the linear approximation holds.

In the following we will neglect these nonlinear effects due to vacuum diffraction upon propagation away from the crystal. If they have to be taken into account, one should replace the response function $G_{0 \mathcal{H}}(18)$ with $X_{0 \mathcal{H}}\left(\xi_{\mathcal{H}}\right)$ (28) and (29) in the equations presented below.

\section{Ultrashort incident pulse with confined wave front}

In the next step, we introduce an incident $\mathrm{x}$-ray pencil beam directed along the unit vector $\hat{\boldsymbol{u}}_{0}(\theta) \equiv \hat{\boldsymbol{u}}_{0}$. The wave front of the pencil beam is bounded in the direction $\hat{\boldsymbol{v}}_{0}(\theta) \equiv \hat{\boldsymbol{v}}_{0}$ perpendicular to $\hat{\boldsymbol{u}}_{0}$ by the transverse profile $\Pi\left(\boldsymbol{v}_{0}\right)$, where $\boldsymbol{v}_{0}=\hat{\boldsymbol{v}}_{0} \boldsymbol{r}$. We assume that the profile has a characteristic width of $\sigma_{v}$ and can be written as a Fourier transform of the angular profile:

$$
\Pi\left(v_{0}\right)=\int_{-\infty}^{\infty} \frac{d \tilde{\theta}}{2 \pi} \Upsilon(\tilde{\theta}) \exp \left[-\mathrm{i} v_{0}(\omega / c)(\tilde{\theta}-\theta)\right]
$$

The characteristic angular spread $\sigma_{\theta}$ in $\Upsilon(\tilde{\theta})$ is related to $\sigma_{v}$ by the uncertainty relationship $\sigma_{\theta} \sigma_{v} \simeq c / \omega$. In particular, for a pencil beam of $\mathrm{x}$ rays with a photon energy $\hbar \omega \simeq 12 \mathrm{keV}(\lambda=2 \pi c / \omega \simeq 0.1 \mathrm{~nm})$ and a lateral spread of $\sigma_{v} \simeq 10 \mu \mathrm{m}$, the angular spread $\sigma_{\theta} \simeq 10^{-5} \mathrm{rad}$.

An ultrashort-in-time incident pencil beam $\mathcal{E}_{\mathrm{i}}(\boldsymbol{r}, t)=$ $\mathcal{E}_{\mathrm{i}} \mathrm{e}^{-\mathrm{i} \omega \tau_{0}} \delta\left(\tau_{0}\right) \Pi\left(v_{0}\right)$ can be presented as a Fourier integral over $\tilde{\theta}$ of the plane-wave components (11) and (12) propagating along directions $\hat{\boldsymbol{u}}_{0}(\tilde{\theta})$ at glancing angles of incidence $\tilde{\theta}$ to the atomic planes around the central angle $\theta$ : 


$$
\begin{aligned}
\mathcal{E}_{\mathrm{i}}(\boldsymbol{r}, t) & =\mathcal{E}_{\mathrm{i}} \int_{-\infty}^{\infty} \frac{d \tilde{\theta}}{2 \pi} \Upsilon(\tilde{\theta}) \mathrm{e}^{-\mathrm{i} \omega \tau_{0}(\tilde{\theta})} \delta\left[\tau_{0}(\tilde{\theta})\right] \\
& \equiv \int_{-\infty}^{\infty} \frac{d \tilde{\theta}}{2 \pi} \Upsilon(\tilde{\theta}) \int_{-\infty}^{\infty} \frac{d \Omega}{2 \pi} \mathrm{e}^{-\mathrm{i}(\omega+\Omega) \tau_{0}(\tilde{\theta})}, \\
\tau_{0}(\tilde{\theta}) & =t-\frac{\hat{\boldsymbol{u}}_{0}(\tilde{\theta}) \boldsymbol{r}_{0}}{c} .
\end{aligned}
$$

The ultrashort-in-time pencil-beam presentation (32) is valid provided the $\tilde{\theta}$ dependence in the delta function $\delta\left[\tau_{0}(\tilde{\theta})\right]$ can be neglected. Since $\tau_{0}(\tilde{\theta}) \simeq \tau_{0}-\frac{v_{0}}{c}(\tilde{\theta}-\theta)$, this is valid if the time delays $\tau_{0}$ we are considering are much longer than the inverse frequency: $\tau_{0} \gg$ $\sigma_{\theta} \sigma_{v} / c \simeq 1 / \omega$.

The spatiotemporal response $\mathcal{E}_{\mathcal{H}}(\boldsymbol{r}, t)$ of the crystal in Bragg diffraction to the excitation by the ultrashort and laterally bound x-ray pulse (32), both for diffracted $(\mathcal{H}=H)$ and forward diffracted $(\mathcal{H}=0)$ components, can now be constructed as a Fourier integral over $\tilde{\theta}$ of the plane-wave solutions (17) and (18):

$$
\begin{aligned}
\mathcal{E}_{\mathcal{H}}(\boldsymbol{r}, t) & =\mathcal{E}_{\mathrm{i}} \int_{-\infty}^{\infty} \frac{d \tilde{\theta}}{2 \pi} \Upsilon(\tilde{\theta}) \mathrm{e}^{-\mathrm{i} \omega(\tilde{\theta}) \tau_{\mathcal{H}}(\tilde{\theta})} G_{0 \mathcal{H}}\left[\xi_{\mathcal{H}}(\tilde{\theta})\right], \\
\tau_{\mathcal{H}}(\tilde{\theta}) & =t-\frac{\hat{\boldsymbol{u}}_{\mathcal{H}}(\tilde{\theta}) \boldsymbol{r}}{c}, \quad \xi_{0}(\tilde{\theta})=\tau_{0}(\tilde{\theta}), \\
\xi_{H}(\tilde{\theta}) & =\tau_{H}(\tilde{\theta})+D(\tilde{\theta}) \frac{\hat{\boldsymbol{v}}_{H}(\tilde{\theta}) \boldsymbol{r}}{c}+\mathcal{T}_{d}(\tilde{\theta}) \delta_{B L} .
\end{aligned}
$$

Here we are using again an important condition that the carrier frequency $\omega(\tilde{\theta})$ satisfies Bragg's law,

$$
\omega(\tilde{\theta}) \sin \tilde{\theta}=H c / 2,
$$

equivalent to (3), and $\alpha=0$ condition.

Since only small $\tilde{\theta}-\theta$ values are significant, we can use $\hat{\boldsymbol{u}}_{\mathcal{H}}(\tilde{\theta})=\hat{\boldsymbol{u}}_{\mathcal{H}}+\delta \hat{\boldsymbol{u}}_{\mathcal{H}}$, with $\delta \hat{\boldsymbol{u}}_{\mathcal{H}}=-\hat{\boldsymbol{v}}_{\mathcal{H}} \delta \tilde{\boldsymbol{\theta}}$. Here, the vectors $\hat{\boldsymbol{v}}_{\mathcal{H}}$ are perpendicular to $\hat{\boldsymbol{u}}_{\mathcal{H}}$ and directed as shown in Figs. 1(a) and 1(b). Applying this result and Taylor expanding $\tau_{\mathcal{H}}(\tilde{\theta})$ and $\omega(\tilde{\theta})$ from (33) to first order in $|\tilde{\theta}-\theta| \ll 1$, we obtain

$$
\begin{array}{rlrl}
\tau_{\mathcal{H}}(\tilde{\theta}) & \simeq \tau_{\mathcal{H}}+\frac{v_{\mathcal{H}}}{c}(\tilde{\theta}-\theta), & v_{\mathcal{H}} \equiv \hat{\boldsymbol{v}}_{\mathcal{H}} \boldsymbol{r}_{\mathcal{H}}, \\
\omega(\tilde{\theta}) & \simeq \omega[1-(\tilde{\theta}-\theta) \cot \theta], & \omega \equiv \omega(\theta), \\
\tau_{\mathcal{H}}(\tilde{\theta}) \omega(\tilde{\theta}) & \simeq \omega \tau_{\mathcal{H}}+\frac{\omega}{c}\left(v_{\mathcal{H}}-\tau_{\mathcal{H}} c \cot \theta\right)(\tilde{\theta}-\theta) .
\end{array}
$$

$G_{0 \mathcal{H}}\left(\xi_{\mathcal{H}}\right)$ is a slowly varying function compared to $\exp \left(-\mathrm{i} \omega \tau_{\mathcal{H}}\right)$. Therefore, one can neglect dependence of $G\left[\xi_{\mathcal{H}}(\tilde{\theta})\right]$ on $\tilde{\theta}$ in performing integration over $\tilde{\theta}$ in (33) provided the lateral shift $v_{\mathcal{H}}$ or/and angular spread $\sigma_{\theta}$ are not too large, so that $\tau_{\mathcal{H}} \gg \sigma_{\theta} v_{\mathcal{H}} / c$. With these assumptions, and using again Eqs. (21)-(23), we arrive at the following general expression for the spatiotemporal dependence of Bragg diffraction from a crystal, excited with an ultrashort-in-time pencil beam with a lateral spatial distribution $\Pi\left(v_{0}\right)$ :

$$
\begin{aligned}
\mathcal{E}_{\mathcal{H}}(\boldsymbol{r}, t) & =\mathcal{E}_{\mathrm{i}} G_{0 \mathcal{H}}\left(\xi_{\mathcal{H}}\right) \Pi\left(\boldsymbol{v}_{\mathcal{H}}-\tau_{\mathcal{H}} c \cot \theta\right) \mathrm{e}^{-\mathrm{i} \omega \tau_{\mathcal{H}},} \\
\tau_{\mathcal{H}} & =t-\frac{\hat{\boldsymbol{u}}_{\mathcal{H}} \boldsymbol{r}}{c}, \quad \mathcal{H}=(0, H), \\
\xi_{0} & =\tau_{0}, \quad \xi_{H}=\tau_{H}+D \frac{\hat{\boldsymbol{v}}_{H} \boldsymbol{r}}{c}+\mathcal{T}_{d} \delta_{B L} .
\end{aligned}
$$

Equations (36) reveal an interesting general property: the spatiotemporal response in Bragg diffraction $(\mathcal{H}=H)$ or in Bragg forward diffraction $(\mathcal{H}=0)$ is given by a product of the corresponding plane-wave spatiotemporal response function $G_{0 \mathcal{H}}\left(\xi_{\mathcal{H}}\right)(18)$ and the spatiotemporal envelope function $\Pi\left(v_{\mathcal{H}}-\tau_{\mathcal{H}} c \cot \theta\right)$, whose peak shifts along $\hat{\boldsymbol{v}}_{\mathcal{H}}$ perpendicular to the optical axis $\hat{\boldsymbol{u}}_{\mathcal{H}}$ linearly in time. Thus, a fixed relationship (36) exists between the time delay of the crystal response and the peak of the lateral shift. In other words, the time delay is mapped onto the lateral spatial shift.

In our previous paper [1], we have shown that the spatial shift takes place in symmetric Bragg diffraction in Bragg scattering geometry. This result of [1] was recently reproduced in [24]. The solution (36) generalizes that result to asymmetric diffraction both in reflection (Bragg) - Fig. 1(a), and transmission (Laue) scattering geometries-Fig. 1(b). This result can be interpreted as follows: the incident wave field with bounded wave front is presented in Eq. (32) as a superposition of plane waves propagating at different angles of incidence. At a different angle, Bragg's law is fulfilled for different photon frequency $\omega(\tilde{\theta})$ (34). As a result, the spatiotemporal response $\mathcal{E}_{\mathcal{H}}(\boldsymbol{r}, t)$ of the crystal in Bragg diffraction (33) is a superposition of wave fields with different carrier frequencies $\omega(\tilde{\theta})(34)$, resulting in a lateral spatial modulation, or, equivalently, in a lateral spatial shift. An alternative interpretation of the derived above general relationship between the time delay and spatial shift is discussed in Appendix C. We will denote this effect as the lateral spatial modulation due to Bragg's law of dispersion, to distinguish it from the spatial modulation due to angular dispersion discussed in Sec. II B, and refer to $\Pi($ ) in Eq. (36) as Bragg's law dispersion envelope.

We conclude: the spatiotemporal response of the crystal to the excitation with an ultrashort and laterally bounded $\mathrm{x}$-ray pulse is accompanied by lateral spatial modulations driven by two different mechanisms: Bragg's law of dispersion and angular dispersion. We will illustrate manifestation of these two mechanisms using particular cases in Secs. III and IV.

Finally, using Eqs. (36) the spatiotemporal dependence of the intensity of Bragg diffraction from a crystal excited with an ultrashort-in-time pencil beam having a lateral spatial distribution $\Pi\left(v_{0}\right)$ can be calculated using

$$
I_{\mathcal{H}}(\boldsymbol{r}, t) \propto\left|\mathcal{E}_{\mathrm{i}}\right|^{2}\left|G_{0 \mathcal{H}}\left(\xi_{\mathcal{H}}\right)\right|^{2} \Pi^{2}\left(v_{\mathcal{H}}-\tau_{\mathcal{H}} c \cot \theta\right) .
$$


We have derived in this section general solutions describing the spatiotemporal response of crystals in Bragg diffraction. In each particular case it is important to know the appropriate plane-wave response functions $G_{0 \mathcal{H}}\left(\xi_{\mathcal{H}}\right)$. They can be calculated numerically in the general case, and examples are discussed in the following Sec. III for the reflection (Bragg) geometry, and in Sec. IV for the transmission (Laue) geometry. In some cases $G_{0 \mathcal{H}}\left(\xi_{\mathcal{H}}\right)$ can be calculated analytically, in particular, for nonabsorbing crystals. In Secs. III and IV we will derive analytical expressions for the response functions of nonabsorbing crystals in the general case of asymmetric diffraction $\eta \neq 0$, in Bragg and Laue scattering geometries, respectively, and perform analysis of the spatiotemporal crystal response using these analytical solutions.

\section{RESPONSE IN BRAGG-CASE GEOMETRY}

\section{A. Diffraction and forward diffraction amplitudes}

We begin this section by summarizing the well-known results of the dynamical theory of x-ray Bragg diffraction for both the forward diffraction $R_{00}$ and diffraction $R_{0 H}$ amplitudes measured at the rear $(z=d)$ and the front $(z=0)$ surfaces of a crystal, respectively:

$$
\begin{gathered}
R_{00}=\mathrm{e}^{\mathrm{i} x_{1} d} \frac{R_{2}-R_{1}}{R_{2}-R_{1} \mathrm{e}^{\mathrm{i}\left(x_{1}-x_{2}\right) d},} \\
R_{0 H}=R_{1} R_{2} \frac{1-\mathrm{e}^{\mathrm{i}\left(x_{1}-x_{2}\right) d}}{R_{2}-R_{1} \mathrm{e}^{\mathrm{i}\left(x_{1}-x_{2}\right) d},},
\end{gathered}
$$

where

$$
\begin{aligned}
\varkappa_{\nu} d & =\chi_{0} \frac{K_{0} d}{2 \gamma_{0}}+\frac{\mathcal{A}}{2} Y_{\nu}(y), \quad R_{\nu}=G Y_{\nu}(y), \\
Y_{\nu}(y) & =\left(-y \pm \sqrt{y^{2}+b /|b|}\right), \quad G=\sqrt{|b| \chi_{H} \chi_{\bar{H}}} / \chi_{\bar{H}} \\
y & =\frac{K_{0} \bar{\Lambda}_{H}}{2 \gamma_{0}}\left[b \alpha+\chi_{0}(1-b)\right], \quad \mathcal{A}=d / \bar{\Lambda}_{H},
\end{aligned}
$$

and

$$
\bar{\Lambda}_{H}=\frac{\sqrt{\gamma_{0}\left|\gamma_{H}\right|}}{\sin \theta} \bar{\Lambda}_{H}^{(\mathrm{s})}, \quad \bar{\Lambda}_{H}^{(\mathrm{s})}=\frac{\sin \theta}{K_{0}|P| \sqrt{\chi_{H} \chi_{\bar{H}}}} .
$$

Here $\chi_{\mathcal{H}}(\mathcal{H}=0, H, \bar{H})$ are Fourier coefficients of the periodic-in-space crystal electric susceptibility $\chi(\boldsymbol{r})$. In general, $\chi_{\mathcal{H}}$ are very small complex parameters. The imaginary part $\mathfrak{s}\left\{\chi_{\mathcal{H}}\right\}$ is related to the cross section of photoabsorption, while the real part $\Re\left\{\chi_{\mathcal{H}}\right\}$ is primarily related to the atomic Thomson scattering amplitude. In many interesting cases, e.g., for Si crystals, $\Re\left\{\chi_{\mathcal{H}}\right\} \gg$ $\Im\left\{\chi_{\mathcal{H}}\right\}$. In certain cases, e.g., for diamond or Be crystals, one can even neglect photoabsorption to a certain extent, and assume $\chi_{\mathcal{H}}$ to be purely real parameters. We make this approximation in the analytic calculations of the response functions below, although as shown by Kato [25] the resulting expressions can often be applied to absorbing perfect crystals by letting the $\chi_{\mathcal{H}}$ be complex if the appropriate branches of square roots, etc. are taken. Typically, $\Re\left\{\chi_{\mathcal{H}}\right\} \sim 10^{-4}-10^{-7}$ for $\mathrm{Si}$ and diamond crystals for 5-20 keV x rays.

The incident and diffracted waves are assumed to be polarized either in the $\sigma$ or the $\pi$ polarization state. The polarization factor in (40) for the $\sigma$-polarization component is $P=1$, and $P=\cos 2 \theta$ for the $\pi$-polarization component, respectively.

The index $\nu=1,2$ identifies two possible solutions for the correction $\varkappa_{\nu}$ of the in-crystal wave vector $\boldsymbol{k}_{0}=$ $\boldsymbol{K}_{0}+x \hat{z}$ with respect to the vacuum wave vector $\boldsymbol{K} 0$.

The diffraction $R_{0 H}$ and forward diffraction $R_{00}$ amplitudes in (38) are essentially functions of one main parameter, the normalized deviation parameter $y$ (39). It contains all the information on the magnitude of the photon frequency $\omega_{0}$, the direction of its momentum $\boldsymbol{K}_{0}$ relative to diffraction vector $\boldsymbol{H}$ and to the internal surface normal $\hat{z}$, the asymmetry factor $b$, and other information pertinent to scattering geometry.

The parameter $\bar{\Lambda}_{H}$ (40) in (39) is an extinction length [26]. In Eq. (40) we also define the extinction length in symmetric scattering geometry $\bar{\Lambda}_{H}^{(\mathrm{s})}$, for which the asymmetry angle $\eta=0$, see Fig. 1(a). In this case $\gamma_{0}=$ $-\gamma_{H}=\sin \theta$, and $\bar{\Lambda}_{H} \rightarrow \bar{\Lambda}_{H}^{(\mathrm{s})}$. An important feature of the symmetric version $\bar{\Lambda}_{H}^{(\mathrm{s})}$ is that it is invariant for a given Bragg reflection, being independent of the photon frequency $\omega_{0}$ or incidence angle $\theta$ [27] to good accuracy for crystals with small photoabsorption; $\bar{\Lambda}_{H}^{(\mathrm{s})}$ is determined solely by the diffraction vector $\boldsymbol{H}$. This can be seen from (40), by using the fact that $\chi_{H} \propto 1 / K_{0}^{2}$ and $\sin \theta \simeq 2 H / K_{0}$. The extinction length $\bar{\Lambda}_{H}^{(\mathrm{s})}$ determines the characteristic interaction length in Bragg diffraction from the atomic planes with diffraction vector $\boldsymbol{H}$. Along with the crystal thickness $d$, the extinction length is another characteristic measure of length in Bragg diffraction.

With the help of the above Eq. (39), and using expression (13) for the deviation parameter $\alpha$, the following relationship can be established between $y$ and $\Omega$ :

$$
\Omega=-\operatorname{sgn}\{b\} \frac{y}{\mathcal{T}_{\Lambda}}+w_{H} \omega,
$$

where

$$
\begin{gathered}
\mathcal{T}_{\Lambda}=\frac{2 \bar{\Lambda}_{H} \sin ^{2} \theta}{c\left|\gamma_{H}\right|} \equiv \mathcal{T}_{\Lambda}^{(\mathrm{s})} \sqrt{|b|} \sin \theta, \\
\mathcal{T}_{\Lambda}^{(\mathrm{s})}=2 \bar{\Lambda}_{H}^{(\mathrm{s})} / c .
\end{gathered}
$$

and

$$
w_{H}=w_{H}^{(\mathrm{s})} \frac{(b-1)}{2 b}, \quad w_{H}^{(\mathrm{s})}=-\frac{\chi_{0}}{2 \sin ^{2} \theta} .
$$


Here, $\mathcal{T}_{\Lambda}$ is the characteristic measure of time in Bragg diffraction associated with the extinction length. It can be directly compared to $\mathcal{T}_{d}=\mathcal{A} \mathcal{T}_{\Lambda}$ (21), another characteristic measure of time in Bragg diffraction associated with the crystal thickness. We have introduced here also the Bragg reflection invariant time constant $\mathcal{T}_{\Lambda}^{(\mathrm{s})}$ (43), which is associated with the Bragg reflection invariant extinction length in symmetric scattering geometry $\bar{\Lambda}_{H}^{(\mathrm{s})}$. Typically, $\bar{\Lambda}_{H}^{(\mathrm{s})} \approx 1-50 \mu \mathrm{m}$ (see Table I in Appendix E), and therefore $\mathcal{T}_{\Lambda}^{(\mathrm{s})} \approx 50-1000 \mathrm{fs}$.

The parameter $w_{H}$ in (44) is a Bragg's law correction due to refraction at the vacuum-crystal interface [18]. Its magnitude $w_{H}^{(\mathrm{s})}$ in symmetric diffraction $(b=-1)$ is a Bragg reflection invariant, similar to the invariance of the extinction length $\bar{\Lambda}_{H}^{(\mathrm{s})}$. While the precise value of $w_{H}^{(\mathrm{s})}$ depends on the Bragg reflection, in most cases it is very small $\left(w_{H}^{(\mathrm{s})} \ll 10^{-4}\right.$, see Table I in Appendix E).

Far off the region of Bragg diffraction, where the deviation parameter $|y| \gg 1$ or equivalently when $\alpha$ (2) is large, the diffraction signal is $R_{0 H}(\infty)=0$ (38), as expected. On the contrary,

$$
R_{00}(\infty)=\mathcal{C}, \quad \mathcal{C}=\exp \left(\mathrm{i} \chi_{0} \frac{K_{0} d}{2 \gamma_{0}}\right)
$$

has a nonzero value that represents the diffraction-free transmission amplitude of the incident radiation with refraction and photoabsorption accounted for by $\mathcal{C}$ through the complex $\chi_{0}$. The actual forward diffraction amplitude is therefore obtained by subtracting off the trivial $y$-independent amplitude $\mathcal{C}(45)$,

$$
\tilde{R}_{00}=R_{00}-\mathcal{C}
$$

resulting in $\tilde{R}_{00}(\infty)=0$. Using the actual forward diffraction amplitude (46), the forward Bragg diffraction response function (18) can be presented as the sum

$$
\begin{aligned}
G_{00}\left(\xi_{0}\right) & =\mathcal{C} \delta\left(\xi_{0}\right)+\tilde{G}_{00}\left(\xi_{0}\right) \\
\tilde{G}_{00}\left(\xi_{0}\right) & =\int_{-\infty}^{\infty} \frac{d \Omega}{2 \pi} \mathrm{e}^{-\mathrm{i} \Omega \xi_{\mathcal{H}}} \tilde{R}_{00}(\omega+\Omega),
\end{aligned}
$$

so that the response is decomposed into the prompt diffraction-free transmission $\mathcal{C} \delta\left(\xi_{0}\right)$, and the delayed actual forward diffraction response function $\tilde{G}_{00}\left(\xi_{0}\right)$. A similar approach has been used in [22] to deal with time dependence of forward resonant scattering from Mössbauer nuclei.

\section{B. Response functions}

The response functions $G_{0 H}\left(\xi_{H}\right)$ and $\tilde{G}_{00}\left(\xi_{0}\right)$ are calculated using Eqs. (18) and (47) with forward diffraction $\tilde{R}_{00}$ and diffraction $R_{0 H}$ amplitudes given by Eqs. (38) and (46) of the previous sections. We use Eq. (41) to perform the integration over $y$ instead of $\Omega$ in the Fourier integrals. In reflection (Bragg) scattering geometry $\gamma_{H}<0$, the asymmetry ratio $b<0$, and therefore the relationship (41) between $\Omega$ and $y$ is actually $\Omega=y / \mathcal{T}_{\Lambda}+w_{H} \omega$.

The response functions $G_{0 H}\left(\xi_{H}\right)$ and $\tilde{G}_{00}\left(\xi_{0}\right)$ can be calculated numerically in the general case. Figure 4 shows examples of such calculations. The left column of Fig. 4 shows examples of reflectivity spectra $\left|R_{0 H}\right|^{2}$ for crystals of different thicknesses $d$. Crystals of rather large thickness are considered $d \gg \bar{\Lambda}_{H}$. In the particular case of the $\boldsymbol{H}=(004)$ Bragg reflection in diamond crystal, the extinction length $\bar{\Lambda}_{H}^{(\mathrm{s})}=3.6 \mu \mathrm{m}$. The reflectivity spectra are nearly rectangular within $|y|<1$, and have an almost crystal thickness independent form and width $\Delta E_{H}$. The reflectivity is almost $100 \%$. Such a high reflectivity is typical for diamond crystals due to low photoabsorption and a high Debye-Waller factor $[33,34]$. The second column in Fig. 4 shows results of calculations for the forward diffraction intensity spectra. They look like inverse diffraction spectra, because of the dominating contribution of the trivial transmission in the diffraction-free region $|y| \gg 1$. The third column shows intensity spectra of the actual forward diffraction. The main contribution is outside the region of the total Bragg reflection. The spectral width $\Delta E_{0} \gg \Delta E_{H}$ and is crystal thickness dependent, varying linearly with $d$.

The last (right) column shows the temporal dependences of the diffraction response function intensity $\left|G_{0 H}\left(\xi_{H}\right)\right|^{2}$, and the actual forward diffraction response functions intensity $\left|\tilde{G}_{00}\left(\xi_{0}\right)\right|^{2}$. In agreement with the behavior of the spectral dependences, $\left|G_{0 H}\left(\xi_{H}\right)\right|^{2}$ is approximately independent of the crystal thickness, while $\left|\tilde{G}_{0 H}\left(\xi_{H}\right)\right|^{2}$ strongly depends on the crystal thickness $d$. The characteristic times of Bragg diffraction $\mathcal{T}_{\Lambda}$ and forward Bragg diffraction $\mathcal{T}_{0}$ are indicated on the graphs. The temporal features at $\xi_{\mathcal{H}}=$ $\mathcal{T}_{d}$ for the crystal with smallest thickness $d=0.05 \mathrm{~mm}$ represent diffracted and forward diffracted wave fields originating from the rear and front surface reflections as schematically illustrated in Fig. 3, cf. also numeric calculations in $[12,18]$. In thicker crystals these echo wave fields arrive at later times, which are outside the presented time range. Interestingly, the characteristic time of the Bragg diffraction response in the range $\xi_{H}>\mathcal{T}_{d}$ changes from $\mathcal{T}_{\Lambda}$ to $\mathcal{T}_{0}$. This reflects the fact that the contribution to this signal comes from the same modes which contribute to forward diffraction, i.e., from the modes propagating through the whole crystal thickness, and not from those propagating only through the extinction length.

More insight can be obtained from analytical solutions. The response functions can be calculated analytically in some specific cases, e.g., in the approximation of a nonabsorbing $\left(\mathfrak{\Im}\left\{\chi_{\mathcal{H}}\right\}=0\right)$ and thick crystal, for which $d \gg \bar{\Lambda}_{H}$, or equivalently $\mathcal{A} \gg 1$. In this case, the diffraction and forward diffraction amplitudes can be approximated by 


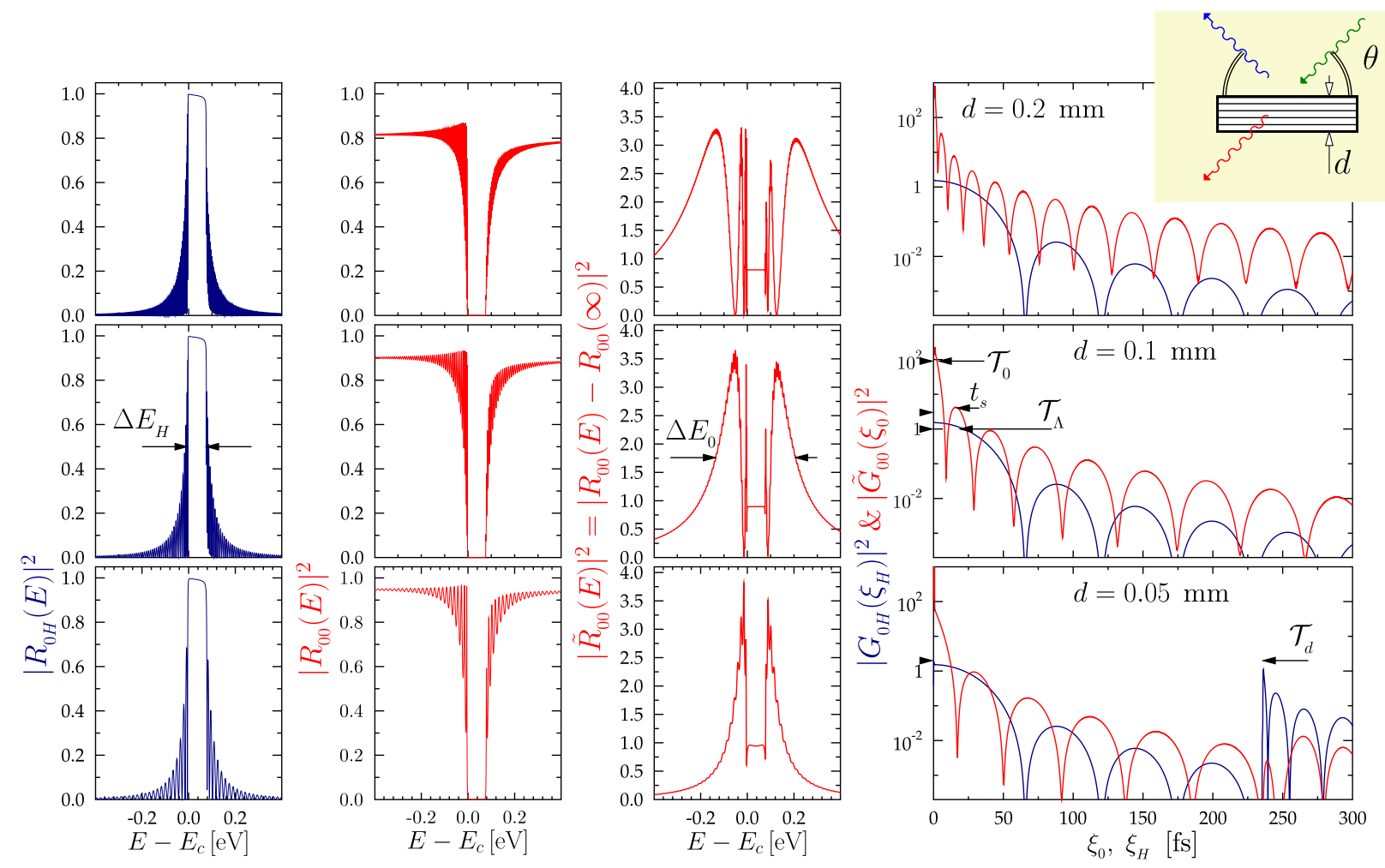

FIG. 4. Spectral dependences (left three columns) of the Bragg diffraction (BD) intensity $\left|R_{0 H}(E)\right|^{2}$, the forward diffraction intensity $\left|R_{00}(E)\right|^{2}$, the actual forward Bragg diffraction (FBD) intensity $\left|\tilde{R}_{00}(E)\right|^{2}=\left|R_{00}(E)-R_{00}(\infty)\right|^{2}$, and the corresponding temporal intensity dependences of the response functions $\left|G_{0 \mathcal{H}}(t)\right|^{2}$ (right column) in symmetric Bragg-case geometry. Numeric calculations use Eqs. (38)-(44), (13), and (18)-(21) for the glancing angle of incidence $\theta=45^{\circ}$ to the (004) reflecting atomic planes in diamond, with the asymmetry angle $\eta=0$. The center of the Bragg reflection region $(y=0)$ corresponds to x-ray photon energy $E_{c}=$ $9.83 \mathrm{keV}$. The spectral and time dependences in diamond crystals of different thickness $d=0.05 \mathrm{~mm}, d=0.1 \mathrm{~mm}$, and $d=0.2 \mathrm{~mm}$ are shown in three different rows from bottom to top, respectively.

$$
\begin{gathered}
R_{0 H}=\mathcal{G} \begin{cases}-y+\mathrm{i} \sqrt{1-y^{2}} & \text { for }|y|<1 \\
-y+\operatorname{sgn}\{y\} \sqrt{y^{2}-1} & \text { for }|y| \geq 1\end{cases} \\
R_{00}=\mathcal{C} \begin{cases}\mathrm{e}^{-\frac{\mathcal{A}}{2}\left(i y+\sqrt{1-y^{2}}\right)} & \text { for }|y|<1 \\
\mathrm{e}^{\mathrm{i} \frac{\mathcal{A}}{2}\left(-y+\operatorname{sgn}\{y\} \sqrt{y^{2}-1}\right)} & \text { for }|y| \geq 1\end{cases}
\end{gathered}
$$

The following observations were used to obtain Eqs. (48) and (49). In the region $y \geqq 1$, both $R_{1} \ll 1$, and $R_{1} / R_{2} \ll 1$, while for $y \leqq-1$, both $R_{2} \ll 1$, and $R_{2} / R_{1} \ll 1$. Neglecting these small terms, Eq. (38) transforms to Eqs. (48) and (49).

Equation (48) represents a well-known result of the dynamical theory, that Bragg diffraction from a nonabsorbing, thick crystal $\mathcal{A} \gg 1$ takes place with total (100\%) reflectivity $\left|R_{0 H}(y)\right|^{2}=1$ within the region $|y|<1$, or equivalently, using (43), within the photon energy range:

$$
\Delta E_{H}=2 \hbar / \mathcal{T}_{\Lambda}
$$

in agreement with results of numeric calculations shown in Fig. 4 (left column). Here $\Delta E_{H}=\hbar \Delta \Omega$.
Using Eqs. (46)-(49), the actual forward diffraction amplitude can be presented in the $|y|>1$ range as

$$
\tilde{R}_{00}=\mathcal{C}\left\{\exp \left[\mathrm{i} \frac{\mathcal{A}}{2}\left(-y+\operatorname{sgn}\{y\} \sqrt{y^{2}-1}\right)\right]-1\right\} .
$$

Equation (51) is in agreement with the results of numeric calculations shown in the third column of Fig. 4. The forward diffraction spectral width

$$
\Delta E_{0} \simeq \Delta E_{H} \frac{\mathcal{A}}{2 \pi}
$$

is a factor of $(\mathcal{A} / 2 \pi)$ broader than the Bragg diffraction spectral width $\Delta E_{H}$, and is crystal thickness dependent.

Using the diffraction amplitude $R_{0 H}$ (48), the forward diffraction amplitude $\tilde{R}_{00}(49)$ and (51), and relationship $\Omega=y / \mathcal{T}_{\Lambda}+w_{H} \omega(42)$, we obtain the plane-wave response functions Eq. (18) in the Bragg-case geometry (see Appendix A for mathematical details):

$$
G_{0 H}\left(\xi_{H}\right)=\mathrm{i} \frac{G}{\mathcal{T}_{\Lambda}} \frac{J_{1}\left(\xi_{H} / \mathcal{T}_{\Lambda}\right)}{\xi_{H} / \mathcal{T}_{\Lambda}} \mathrm{e}^{-\mathrm{i} w_{H} \omega \xi_{H}}
$$



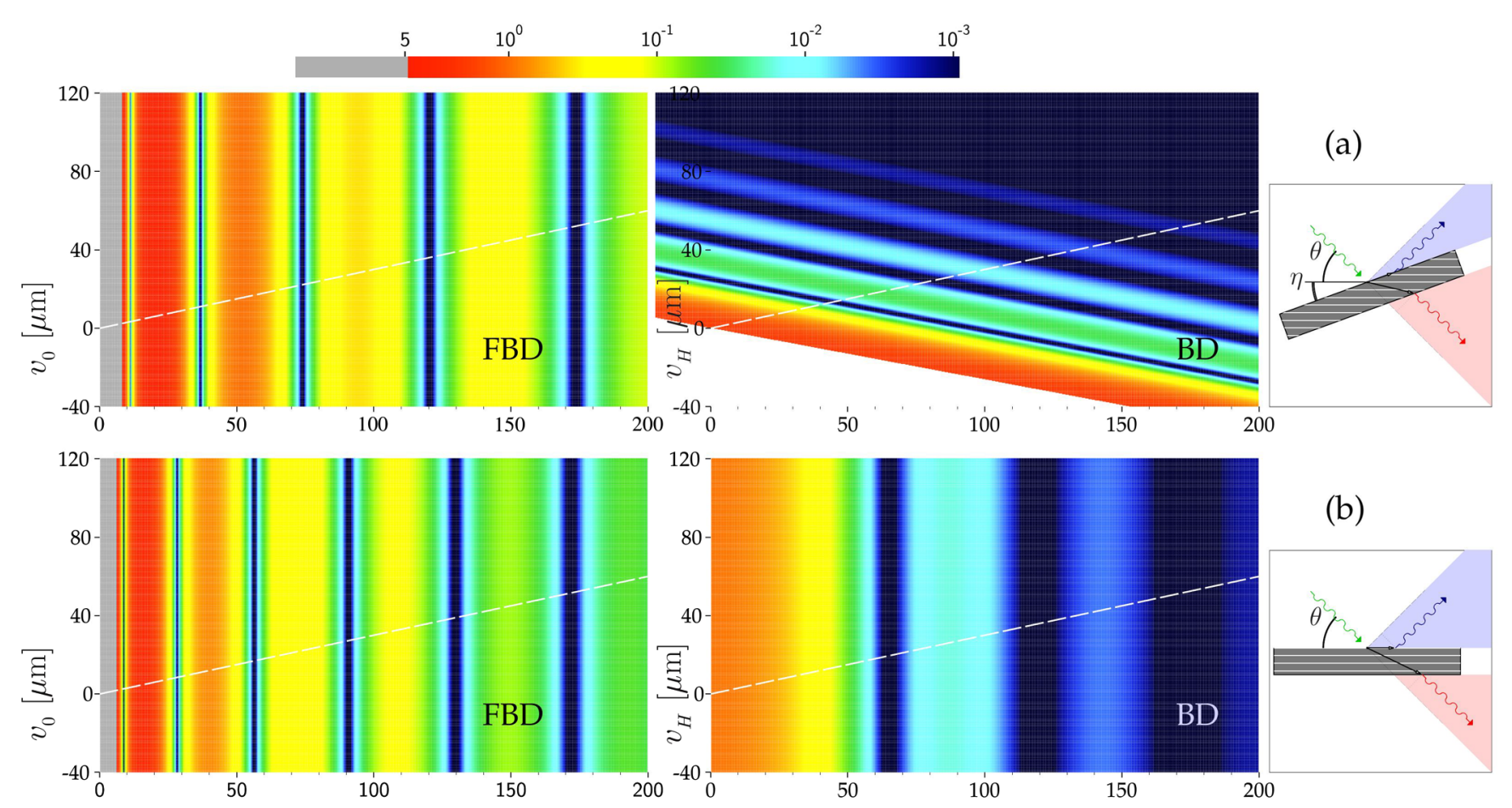

(b)
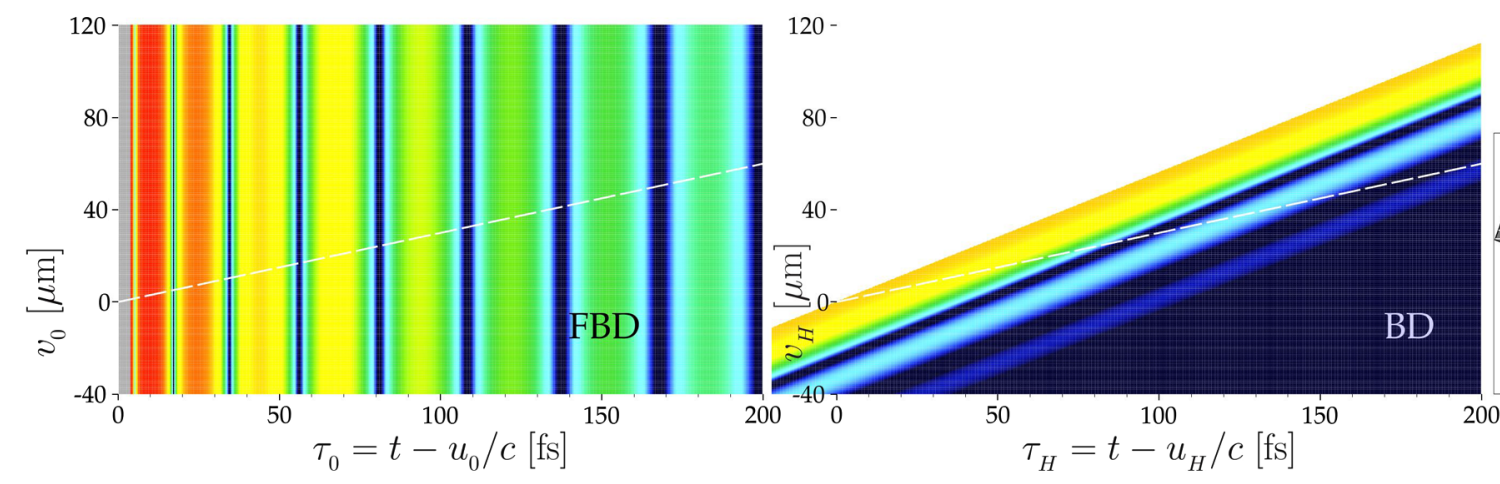

(c)

FIG. 5. Spatiotemporal intensity profiles of FBD and BD from a $100 \mu \mathrm{m}$ thick diamond crystal from the (004) Bragg reflection $\left(\bar{\Lambda}_{H}^{(s)}=3.6 \mu \mathrm{m}\right)$, in the reflection (Bragg) scattering geometry-Fig. 1(a)—with asymmetry angles $\eta=20^{\circ}$ (a), $\eta=0^{\circ}(\mathrm{b})$, and $\eta=-20^{\circ}$ (c). We plot Eq. (37) using the plane-wave response functions (53) and (54) and a Gaussian lateral spatial profile of the incident x-ray beam with $\sigma_{v}=1000 \mu \mathrm{m}$ (i.e., a practically unbounded incident wave front). Other parameters: $\theta=45^{\circ}$, $E=9.8 \mathrm{keV}$, which are the same as those used for the calculations of the response functions shown in Fig. 4. The intensity front tangent in Bragg diffraction (BD) is $d v_{\mathcal{H}} / d \tau_{\mathcal{H}}=-c / D(22)$. White dashed lines are traces of the Bragg's law dispersion envelopes $\Pi^{2}\left(v_{\mathcal{H}}-\tau_{\mathcal{H}} c \cot \theta\right)$ (36) and (37) with a tangent $d v_{\mathcal{H}} / d \tau_{\mathcal{H}}=c \cot \theta$, cf. Fig. 6.

$$
\begin{gathered}
\tilde{G}_{00}\left(\xi_{0}\right)=-\frac{\mathcal{C}}{2 \mathcal{T}_{0}} \frac{J_{1}\left[\sqrt{\frac{\xi_{0}}{\mathcal{T}_{0}}\left(1+\frac{\xi_{0}}{\mathcal{T}_{d}}\right)}\right]}{\sqrt{\frac{\xi_{0}}{\mathcal{T}_{0}}\left(1+\frac{\xi_{0}}{\mathcal{T}_{d}}\right)}} \mathrm{e}^{-\mathrm{i} w_{H} \omega \xi_{0}}, \\
\mathcal{T}_{0}=\mathcal{T}_{\Lambda} / \mathcal{A} \equiv \frac{2\left[\bar{\Lambda}_{H}^{(\mathrm{s})}\right]^{2}}{c\left(d / \gamma_{0}\right)}
\end{gathered}
$$

These solutions are valid if $\xi_{\mathcal{H}}<\mathcal{T}_{d}(\mathcal{H}=0, H)$, i.e., over the duration of time that is less than the total propagation time through the crystal $\mathcal{T}_{d}$. Thus, this solution does not include possible reflections from the rear and front crystal surfaces.

In the limit of symmetric Bragg scattering, the response function envelopes are the same as those obtained in [1]. In

general, however, an asymmetric geometry changes the characteristic time constants.

According to Eq. (53), the characteristic time constant in Bragg diffraction is $\mathcal{T}_{\Lambda}$ (42), which is a function of the asymmetry factor $b$; in fact, it scales with $\sqrt{|b|}$. By appropriately choosing the asymmetry factor $b$, the time response can be made faster or slower compared to the time response of Bragg diffraction in symmetric geometry. Additionally, the uncertainty-type relationship (50) associates the characteristic time constant of diffraction with its spectral width. As a consequence of Eqs. (50) and (42), a well-known result of the dynamical theory can be reproduced: the Bragg reflection spectral width scales with $1 / \sqrt{|b|}, \Delta E_{H}=\hbar c /\left(\bar{\Lambda}_{H}^{(\mathrm{s})} \sqrt{|b|} \sin \theta\right)$. Note that both 

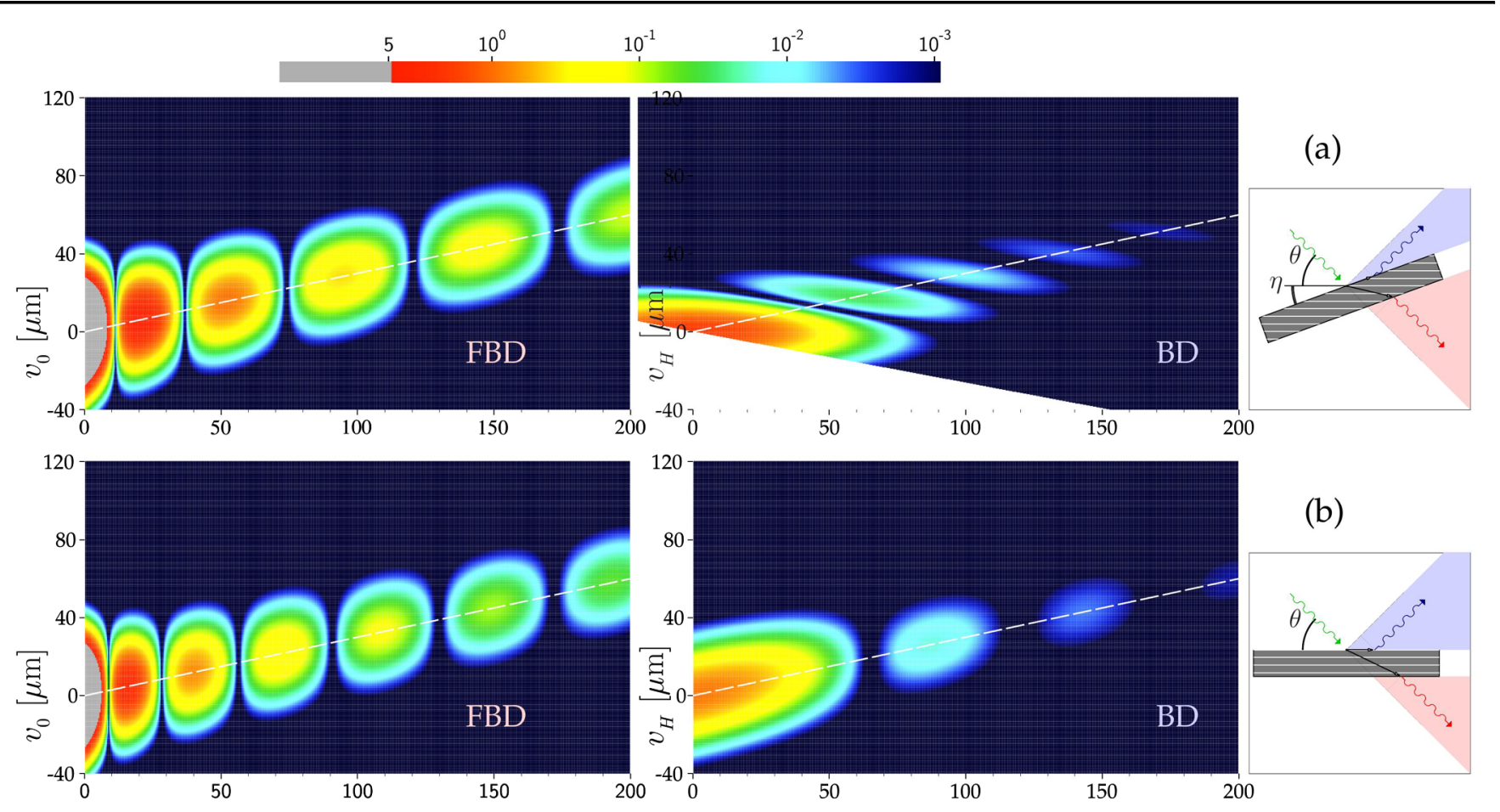

100

150

(b)
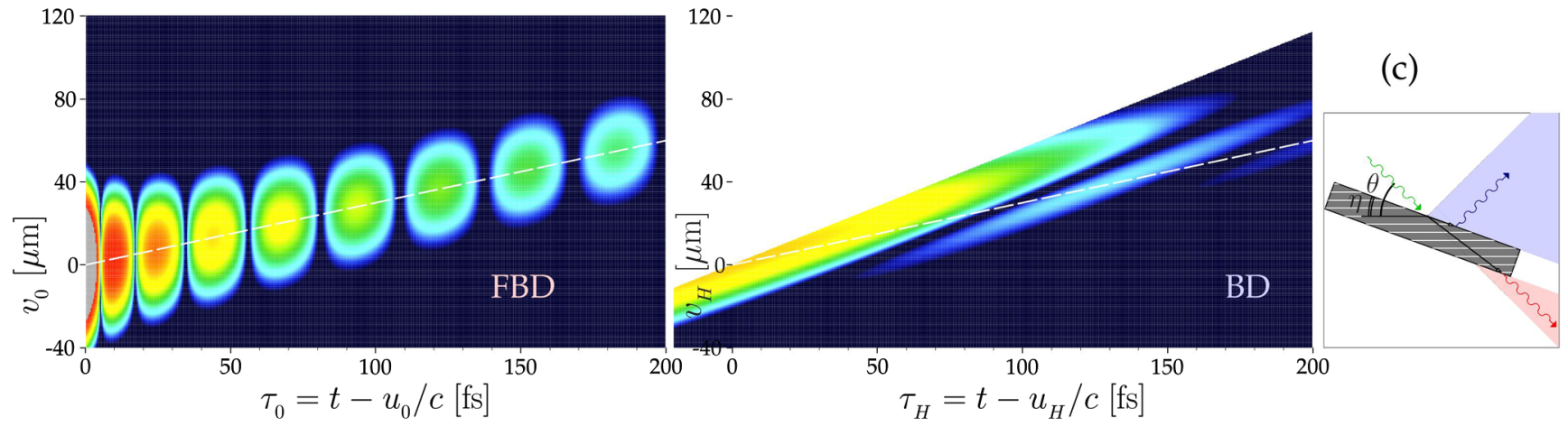

FIG. 6. Spatiotemporal intensity profiles of FBD and BD for similar parameters as that of Fig. 5. Here, however, the incident $\mathrm{x}$ rays have a bounded lateral spatial profile $\Pi\left(v_{0}\right)$ that is assumed to be Gaussian with $\sigma_{v}=10 \mu \mathrm{m}$. Because of this, the spatiotemporal intensity profiles shown in Fig. 5 are limited now by a tight Bragg's law dispersion envelope $\Pi^{2}\left(v_{\mathcal{H}}-\tau_{\mathcal{H}} c \cot \theta\right)(37)$ with a tangent $d v_{\mathcal{H}} / d \tau_{\mathcal{H}}=c \cot \theta$, shown as white dashed line.

the typical energy and time scales are predominantly determined by a single parameter, namely, the extinction length $\bar{\Lambda}_{H}^{(\mathrm{s})}$. This fact explains why Bragg diffraction is not instantaneous, as it builds by multiple, coherent scattering of $x$ rays within the extinction length.

The characteristic time constant of forward Bragg diffraction is significantly different, since different multiple scattering processes are involved. According to Eqs. (54) and (55), the characteristic time is given by $\mathcal{T}_{0}$, which is a factor of $\mathcal{A}=d / \bar{\Lambda}_{H}$ smaller than the characteristic constant of Bragg diffraction $\mathcal{T}_{\Lambda}$. Interestingly, $\mathcal{T}_{0}$ is practically the same as in symmetric scattering geometry. In the general case, it is basically defined by the Bragg reflection invariant $\bar{\Lambda}_{H}^{(\mathrm{s})}$ and the effective crystal thickness seen by incident $\mathrm{x}$ rays $d / \gamma_{0}$. From Eq. (54), we also calculate that the first trailing maximum of the forward diffraction response function appears at $t_{s}=26 \mathcal{T}_{0}$, as illustrated in Fig. 4, and its duration is $\Delta t_{s}=16.5 \mathcal{T}_{0}$.

We note also that, if $\xi_{0} \ll \mathcal{T}_{d}$, the expression for the forward diffraction response function given in Eqs. (54) and (55) can be simplified to

$$
\tilde{G}_{00}\left(\xi_{0}\right)=-\frac{\mathcal{C}}{2 \mathcal{T}_{0}} \frac{J_{1}\left(\sqrt{\xi_{0} / \mathcal{T}_{0}}\right)}{\sqrt{\xi_{0} / \mathcal{T}_{0}}} \mathrm{e}^{-\mathrm{i} w_{H} \omega \xi_{0}}
$$

\section{Analysis of the spatiotemporal response in Bragg-case geometry}

By combining the analytical expressions for the planewave response functions (53) and (54) obtained in the previous section with the general solutions (36) and (37), 
we are now in a position to describe the spatiotemporal response of crystals in x-ray Bragg diffraction resulting from the excitation by an ultrashort and laterally confined $\mathrm{x}$-ray pulse.

To make the analysis more instructive, we show in Figs. 5 and 6 examples of $2 \mathrm{D}\left(\tau_{\mathcal{H}}, v_{\mathcal{H}}\right)$ color plots of the spatiotemporal intensity profiles of forward Bragg diffraction (FBD) and Bragg diffraction (BD) from a $100 \mu \mathrm{m}$ thick diamond crystal in the (004) Bragg reflection $\left(\bar{\Lambda}_{H}^{(\mathrm{s})}=3.6 \mu \mathrm{m}\right)$ with the asymmetry angle $\eta=20^{\circ}$ (a), $\eta=0^{\circ}$ (b), or $\eta=-20^{\circ}$ (c).

Figure 5 shows examples of calculations that apply the above-mentioned equations to an incident wave front that is, for all practical purposes, laterally unbounded (we assume that the incident spatial profile has a Gaussian distribution with $\sigma_{v}=1000 \mu \mathrm{m}$ ). In the symmetric caseFig. 5(b) - the spatiotemporal profiles of both FBD and BD are homogeneous in the lateral spatial shift $v_{\mathcal{H}}$, i.e., they show no variation along the plane perpendicular to the appropriate optical axis $\hat{\boldsymbol{u}}_{\mathcal{H}}$ - see Fig. 1(a). The FBD response remains independent of $v_{\mathcal{H}}$ for nonzero values of the asymmetry angle $\eta \neq 0$, as the plots demonstrate in the left columns of Figs. 5(a) and 5(c). In contrast, the BD profiles acquire modulations along $v_{H}$ if $\eta \neq 0$. They also produce the impression that the wave fronts of the BD wave fields are inclined. There are two phase factors in the expression for the wave field $\mathcal{E}_{H}(\boldsymbol{r}, t)$ in Eq. (36). The first is $\exp \left(-\mathrm{i} \omega \tau_{\mathcal{H}}\right)$ defining the wave front perpendicular to the optical axis $\hat{\boldsymbol{u}}_{H}$, and another one $\exp \left(-\mathrm{i} w_{H} \omega \xi_{H}\right)$ resulting from the plane-wave response function $G_{0 H}\left(\xi_{H}\right)$ (53). Since the second contribution is due to a small refractive correction, the wave front is practically not inclined. The pronounced effect seen in Figs. 5(a) and 5(c) is actually the inclined amplitude (intensity) front due to the amplitude modulation perpendicular to $\hat{\boldsymbol{u}}_{H}$ resulting from angular dispersion, discussed in Sec. II B. Formally, the inclination and modulation reveal themselves through the argument $\xi_{H}$ of $G_{0 H}\left(\xi_{H}\right)$ which depends both on time $t$, and, if $\eta \neq 0$, also on the space variable $v_{H}$-Eq. (23). The magnitude of the inclination to $\hat{\boldsymbol{u}}_{H}$ is $D / c$, it scales with the normalized angular dispersion rate $D$. The inclination of the intensity front changes sign with the sign of $\eta$. The tilting of the intensity profiles due to Bragg diffraction was previously noted by Bushuev [20].

In all cases, varying the magnitude and sign of the asymmetry angle $\eta$ changes the time constants $\mathcal{T}_{0}$ and $\mathcal{T}_{\Lambda}$, resulting in either dilation-Fig. 5(a)—or contractionFig. 5(c)—of the oscillating intensity structures associated with the spatiotemporal response; this dilation or contraction as compared to the symmetric case occurs both in the $v_{\mathcal{H}}$ and the $\tau_{\mathcal{H}}$ directions, as shown in Fig. 5(b).

In the next step, we narrow considerably the lateral spatial profile of the incident x-ray beam. Figure 6 shows examples of calculations for incident $\mathrm{x}$ rays having a Gaussian lateral spatial profile with $\sigma_{v}=10 \mu \mathrm{m}$, with all other parameters being identical to those in Fig. 5 . The wave field in the direction perpendicular to the optical axis $\hat{\boldsymbol{u}}_{H}$ is bounded by the Bragg's law dispersion envelope $\Pi^{2}\left(v_{\mathcal{H}}-\tau_{\mathcal{H}} c \cot \theta\right)$-Eqs. (36) and (37). White dashed lines in Figs. 5 and 6 are traces of the envelope. The tangent $d v_{\mathcal{H}} / d \tau_{\mathcal{H}}=c \cot \theta$ is independent on whether the geometry is symmetric $\eta=0$-Fig. 6(b), or asymmetric $\eta \neq 0$-Figs. 6(a) and 6(c). As has been mentioned in Sec. II C this is a result of Bragg's law dispersion due to angular spread in the incident beam caused by the bounded wave front.

The two effects of lateral amplitude modulation of the wave field, due both to angular dispersion and to Bragg's law of dispersion, can be clearly distinguished by comparing the spatiotemporal profiles in Fig. 5 and in Fig. 6.

\section{RESPONSE IN LAUE-CASE GEOMETRY}

\section{A. Diffraction, forward diffraction amplitudes, and response functions}

The wave field amplitudes in transmission (Laue) geometry are given by the following expressions [6-11]:

$$
\begin{aligned}
R_{00} & =\frac{1}{R_{2}-R_{1}}\left(R_{2} \mathrm{e}^{\mathrm{i} x_{1} d}-R_{1} \mathrm{e}^{\mathrm{i} \varkappa_{2} d}\right), \\
R_{0 H} & =\frac{R_{1} R_{2}}{R_{2}-R_{1}}\left(\mathrm{e}^{\mathrm{i} \varkappa_{1} d}-\mathrm{e}^{\mathrm{i} \varkappa_{2} d}\right) .
\end{aligned}
$$

The notation is the same as in (39), but in contrast to the reflection (Bragg) geometry, the asymmetry factor $b$ (39) is positive in transmission geometry. Using Eqs. (39) and (44), the wave field amplitudes (57) can be presented as

$$
\begin{aligned}
R_{00} & =\mathcal{C} \mathrm{e}^{-\mathrm{i} \mathcal{A} y / 2} W(y), \\
W(y) & =\cos \left(\frac{\mathcal{A}}{2} \sqrt{y^{2}+1}\right)+\mathrm{i} y \frac{\sin \left(\frac{\mathcal{A}}{2} \sqrt{y^{2}+1}\right)}{\sqrt{y^{2}+1}}, \\
R_{0 H} & =\mathrm{i} \mathcal{C} G \mathrm{e}^{-\mathrm{i} \mathcal{A} y / 2} V(y), \quad V(y)=\frac{\sin \left(\frac{\mathcal{A}}{2} \sqrt{y^{2}+1}\right)}{\sqrt{y^{2}+1}} .
\end{aligned}
$$

Using the forward diffraction amplitude $R_{00}$ given by (58), the diffraction amplitude $R_{0 H}$ given by (59), the relationship $\Omega=-y / \mathcal{T}_{\Lambda}+w_{H} \omega$ (42), and assuming zero photoabsorption, we compute the plane-wave response functions Eq. (18) for the Laue case which are given by (see Appendix B for mathematical details)

$$
\begin{gathered}
G_{00}\left(\xi_{0}\right)=\tilde{G}_{00}\left(\xi_{0}\right)+\mathcal{C} \delta\left(\xi_{0}\right) \\
\tilde{G}_{00}\left(\xi_{0}\right)=\frac{\mathcal{C}}{2 \mathcal{T}_{0}}\left(1-\frac{\xi_{0}}{\mathcal{T}_{d}}\right) \frac{J_{1}\left[\sqrt{\frac{\xi_{0}}{\mathcal{T}_{0}}\left(1-\frac{\xi_{0}}{\mathcal{T}_{d}}\right)}\right]}{\sqrt{\frac{\xi_{0}}{\mathcal{T}_{0}}\left(1-\frac{\xi_{0}}{\mathcal{T}_{d}}\right)}} \mathrm{e}^{-\mathrm{i} w_{H} \omega \xi_{0}} \\
{\left[0<\xi_{0}<\mathcal{T}_{d}\right]}
\end{gathered}
$$



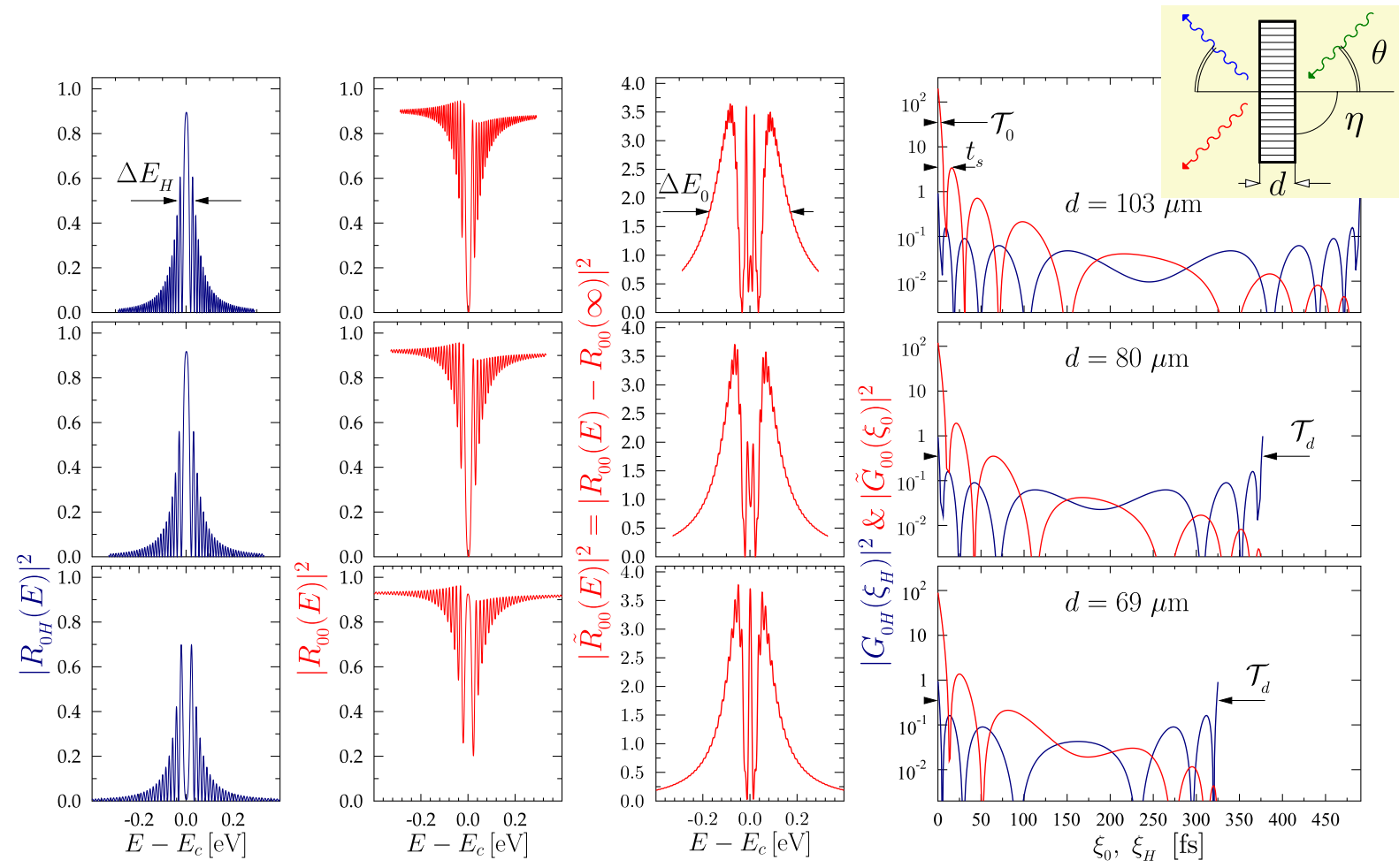

FIG. 7. Spectral dependences (left three columns) of the Bragg diffraction (BD) intensity $\left|R_{0 H}(E)\right|^{2}$, the forward Bragg diffraction intensity $\left|R_{00}(E)\right|^{2}$, the actual forward Bragg diffraction (FBD) intensity $\left|\tilde{R}_{00}(E)\right|^{2}=\left|R_{00}(E)-R_{00}(\infty)\right|^{2}$, and the corresponding temporal intensity dependences of the response functions $\left|G_{0 \mathcal{H}}\left(\xi_{\mathcal{H}}\right)\right|^{2}$ (right column) in Laue-case geometry. Numeric calculations use Eqs. (57), (39)-(44), and (18) for the glancing angle of incidence $\theta=45^{\circ}$ to the (004) reflecting atomic planes in diamond, with the asymmetry angle $\eta=90^{\circ}$. The center of the Bragg reflection region $(y=0)$ corresponds to the x-ray photon energy $E_{\mathrm{c}}=$ $9.83 \mathrm{keV}$. The spectral and time dependences in diamond crystals of different thickness $d=67 \mu \mathrm{m}, d=80 \mu \mathrm{m}$, and $d=104 \mu \mathrm{m}$ are shown in three different rows from bottom to top, respectively.

$$
\begin{aligned}
G_{0 H}\left(\xi_{H}\right)=- & \mathrm{i} \frac{\mathcal{C G}}{2 \mathcal{T}_{\Lambda}} J_{0}\left[\sqrt{\frac{\xi_{H}}{\mathcal{T}_{0}}\left(1-\frac{\xi_{H}}{\mathcal{T}_{d}}\right)}\right] \mathrm{e}^{-\mathrm{i} w_{H} \omega \xi_{H}} \\
& {\left[0<\xi_{H}<\mathcal{T}_{d}\right] . }
\end{aligned}
$$

Here we use characteristic time constants defined previously: $\mathcal{T}_{d}$ in Eq. (21), $\mathcal{T}_{\Lambda}$ in Eq. (43), and $\mathcal{T}_{0}$ in Eq. (55). Both Eqs. (60) and (61) can be simplified if $\xi_{\mathcal{H}} \ll \mathcal{T}_{d}$

$$
\begin{gathered}
\tilde{G}_{00}\left(\xi_{0}\right)=\frac{\mathcal{C}}{2 \mathcal{T}_{0}} \frac{J_{1}\left(\sqrt{\xi_{0} / \mathcal{T}_{0}}\right)}{\sqrt{\xi_{0} \mathcal{T}_{0}}} \mathrm{e}^{-\mathrm{i} w_{H} \omega \xi_{0}}, \\
G_{0 H}\left(\xi_{H}\right)=-\mathrm{i} \frac{\mathcal{C} G}{2 \mathcal{T}_{\Lambda}} J_{0}\left(\sqrt{\xi_{H} / \mathcal{T}_{0}}\right) \mathrm{e}^{-\mathrm{i} w_{H} \omega \xi_{H}}
\end{gathered}
$$

Remarkably, for small $\xi_{0} \equiv \tau_{0} \ll \mathcal{T}_{d}$, the forward diffraction plane-wave response function $\tilde{G}_{00}\left(\xi_{0}\right)$ in the Laue-case geometry (63), and its counterpart (56) in the Bragg-case geometry, are equivalent, however, with inverted signs, as a consequence of $\operatorname{sgn}\{b\}$ in Eq. (41).

The time constant $\mathcal{T}_{0}$ (55) is essentially the same in both transmission (Laue) and reflection (Bragg) geometries.
It equals the time constant $\mathcal{T}_{\Lambda}^{(\mathrm{s})}$ in symmetric Bragg diffraction scaled by a ratio $\bar{\Lambda}_{H}^{(\mathrm{s})} /\left(d / \gamma_{0}\right)$ of the symmetric extinction length $\bar{\Lambda}_{H}^{(\mathrm{s})}$ to the effective crystal thickness $\left(d / \gamma_{0}\right)$, i.e., he crystal thickness seen by incident $x$ rays. The time constant of forward Bragg diffraction $\mathcal{T}_{0}(55)$ is thus a universal parameter for all symmetric or asymmetric, transmission or reflection scattering geometries. It is the parameter controlling the strength and duration of forward Bragg diffraction response, with the extinction length and the effective crystal thickness being the primary variables composing $\mathcal{T}_{0}$.

\section{B. Analysis of the spatiotemporal response}

Expressions (60) and (61) for the plane-wave response functions are very similar to the analogous expressions obtained by Graeff and Malgrange in [15,16], with the exception that $G_{00}\left(\xi_{0}\right)$ in (60) contains also the prompt $\delta$-function contribution. It originates from the spectral components in the incident pulse with frequencies far from the Bragg diffraction region that propagate diffraction-free through the crystal. In Appendix B we also provide some more details on the comparison with the results of $[15,16]$.

Figure 7 shows results of numeric calculations of the plane-wave response function intensities $\left|G_{0 \mathcal{H}}\left(\xi_{H}\right)\right|^{2}$ and related to them spectral dependences $\left|R_{0 \mathcal{H}}(E)\right|^{2}$ for 

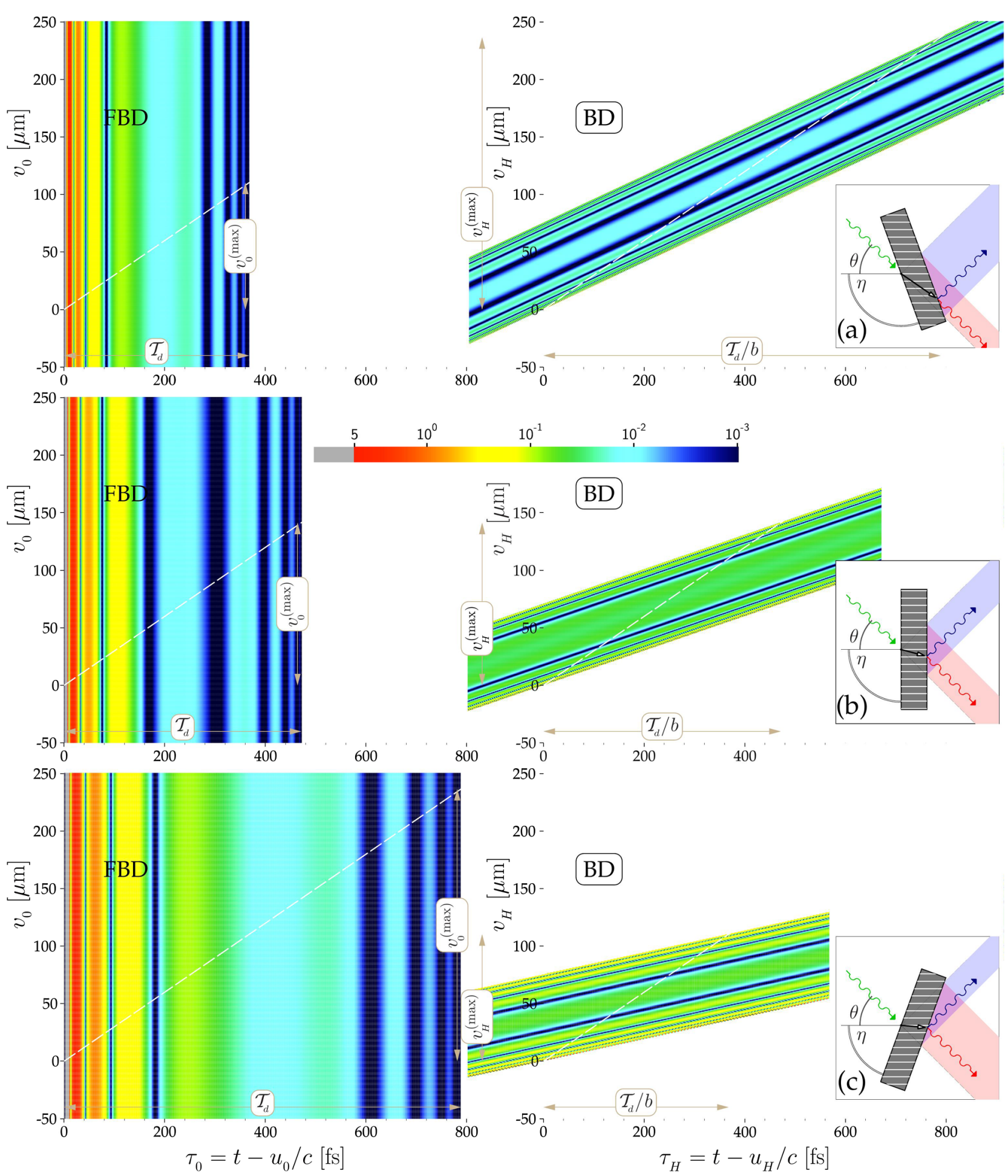

$200-$
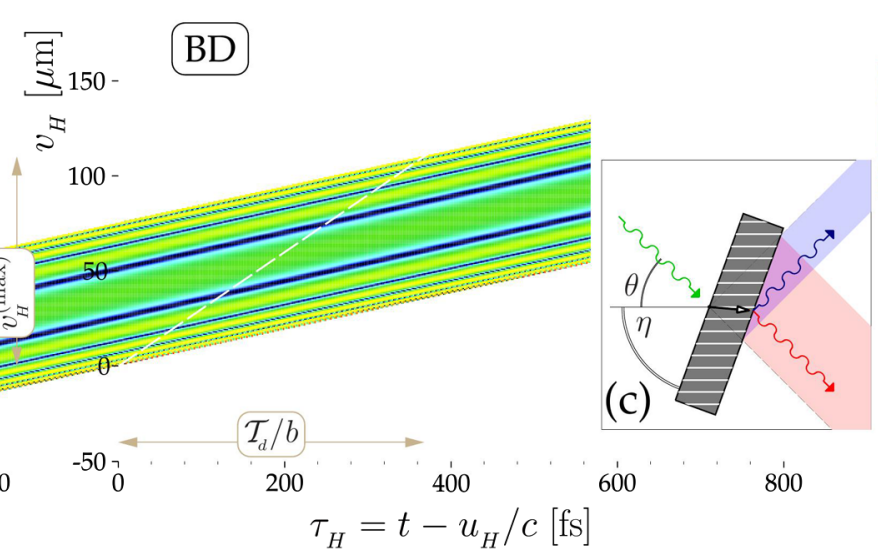

800

FIG. 8. Spatiotemporal intensity profiles of FBD and BD from a $100 \mu \mathrm{m}$ thick diamond crystal from the (004) Bragg reflection $\left(\bar{\Lambda}_{H}^{(s)}=3.6 \mu \mathrm{m}\right)$, in the transmission (Laue) scattering geometry-Fig. 1 (b) - with asymmetry angles $\eta=110^{\circ}$ (a), $\eta=90^{\circ}$ (b), and $\eta=70^{\circ}$ (c). We plot Eq. (37) with the plane-wave response functions (60) and (61) and a Gaussian lateral spatial profile of the incident x-ray beam with $\sigma_{v}=1000 \mu \mathrm{m}$, (i.e., a practically unbounded incident wave front). Other parameters: $\theta=45^{\circ}, E=$ $9.8 \mathrm{keV}$, which are the same as those used for response functions shown in Fig. 7. The intensity front tangent in Bragg diffraction (BD) is $d v_{\mathcal{H}} / d \tau_{\mathcal{H}}=-c / D(22)$. White dashed lines are traces of the Bragg's law dispersion envelopes $\Pi^{2}\left(v_{\mathcal{H}}-\tau_{\mathcal{H}} c \cot \theta\right)(36)$ and (37) with a tangent $d v_{\mathcal{H}} / d \tau_{\mathcal{H}}=c \cot \theta$, cf. Fig. 9. 

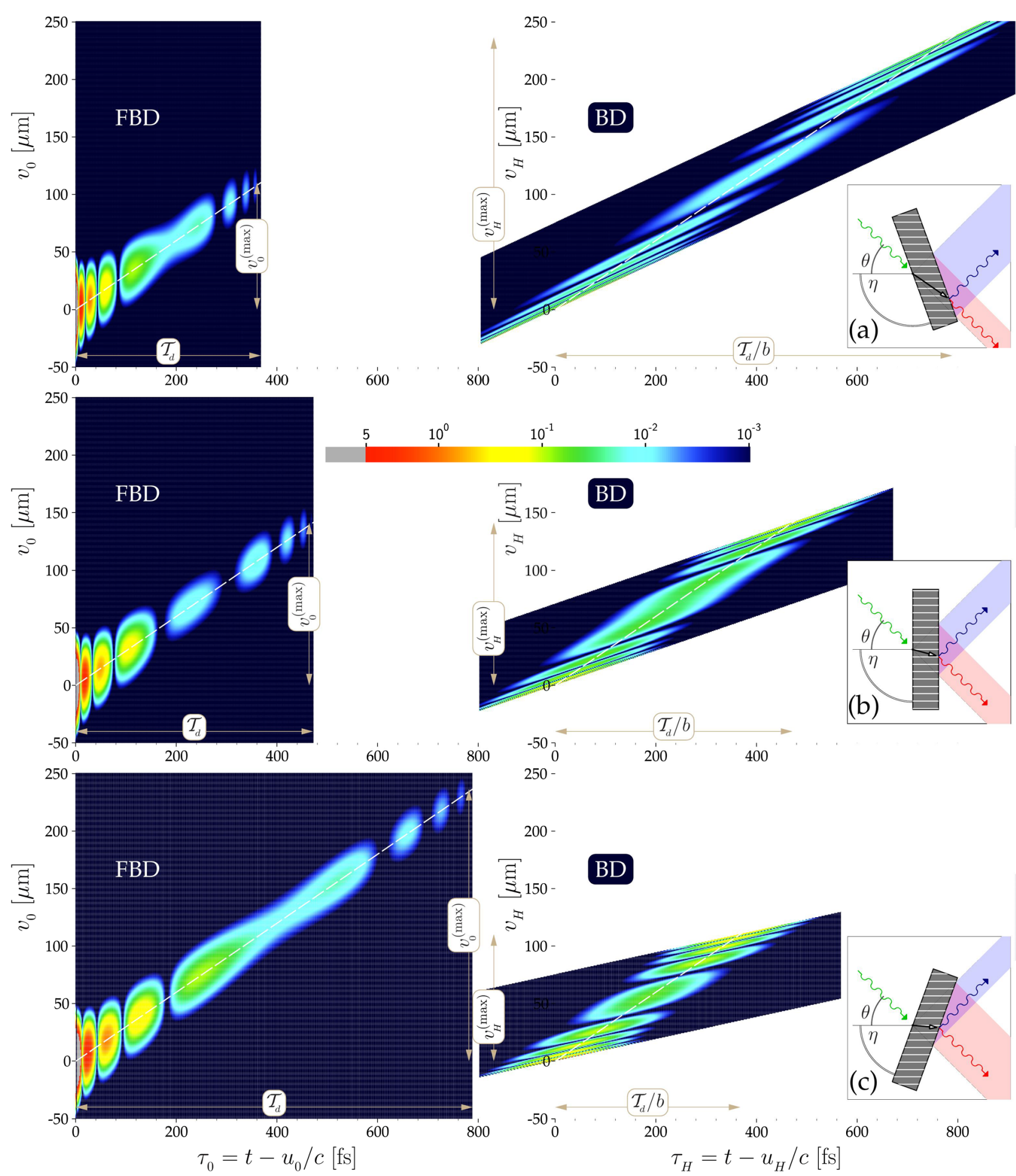

$200-$

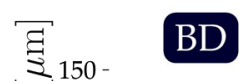

FIG. 9. Spatiotemporal intensity profiles of FBD and BD for similar parameters as that of Fig. 8. Here, however, the incident $\mathrm{x}$-rays have a bounded lateral spatial profile $\Pi\left(v_{0}\right)$ that is assumed to be Gaussian with $\sigma_{v}=10 \mu \mathrm{m}$. Because of this, the spatiotemporal intensity profiles shown in Fig. 8 are limited now by a tight Bragg's law dispersion envelope $\Pi^{2}\left(v_{\mathcal{H}}-\tau_{\mathcal{H}} c \cot \theta\right)(37)$ with a tangent $d v_{\mathcal{H}} / d \tau_{\mathcal{H}}=c \cot \theta$, shown as white dashed line.

the Laue case. These calculations are provided to facilitate "visualization" of the analytical solutions given by Eqs. (57)-(61). The dependences shown in Fig. 7 are counterparts of the analogous Bragg-case dependences shown in Fig. 4. They are calculated under the same conditions, with the single difference being that the asymmetry angle is now $\eta=\pi / 2$ ("symmetric" Laue case), instead of $\eta=0$ in Fig. 4 (symmetric Bragg case). 
In the Laue case, the spectral range where Bragg diffraction takes place scales with $\Delta E_{H}$. While this is similar to the Bragg-case geometry, for Laue there is no region of total reflection. The dominant feature of the spectral intensity dependence in Fig. 7 is the intensity oscillations, which is associated with the well-known Pendellösung effect of Ewald [4]. Pendellösung is basically related to oscillations of the energy flow between the forward diffracted and diffracted beams in the Laue-case geometry. The period of oscillations scales with the extinction length $\bar{\Lambda}_{H}$, which is $3.6 \mu \mathrm{m}$ in this particular case. For the calculations presented in the middle row the crystal thickness $d$ is reduced by $2 \pi \bar{\Lambda}_{H}=22.6 \mu \mathrm{m}$, compared to the $d$ value used in the calculations presented in the top row. The crystal thickness $d$ is further decreased by $\pi \bar{\Lambda}_{H}=11.3 \mu \mathrm{m}$ for the calculations presented in the bottom row. Varying the crystal thickness leads to periodic in $d$ and in $E$ variations of the intensities that are complementary for the diffracted and forward diffracted signals. Interestingly, in the spectral range $\left|E-E_{\mathrm{c}}\right|>\Delta E_{H}$, the actual forward diffraction intensity $\left|\tilde{R}_{00}(E)\right|^{2}=\left|R_{00}(E)-R_{00}(\infty)\right|^{2}$ has a structure very similar to that in the Bragg-case geometry, cf. Fig. 7. This is in agreement with the fact that $\tilde{G}_{00}\left(\xi_{0}\right)$ for small $\xi_{0}$ values are identical (modulo the inverted sign) in Braggcase and Laue-case geometries, cf. Eqs. (56) and (62).

Comparison of Eqs. (61) and (53), as well as the results of numeric calculations of $\left|G_{0 H}\left(\xi_{H}\right)\right|^{2}$ in Figs. 7 and 4, show that the characteristic time of diffraction in Laue-case geometry is $\mathcal{T}_{0}$ (53), i.e., different from the characteristic time of diffraction $\mathcal{T}_{\Lambda}$ (43) in Bragg-case geometry. This evidences that two different characteristic length scales are involved for these two different diffraction cases.

Equations (60) and (61) and Fig. 7 demonstrate a signature feature of the Laue-case plane-wave response functions $G_{0 \mathcal{H}}\left(\xi_{\mathcal{H}}\right)$. Unlike the Bragg-case analogs, $G_{0 \mathcal{H}}\left(\xi_{\mathcal{H}}\right)$ vanish outside the range $0>\xi_{\mathcal{H}}>\mathcal{T}_{d}$. This effect has been reported and discussed by Shastri et al. $[12,13]$ using numeric calculations, and by Graeff and Malgrange using analytical solutions in $[15,16]$.

This feature, however, deserves a more detailed discussion, as it is in fact valid only under certain conditions, but not in general. To illustrate this, we refer to the example $2 \mathrm{D}\left(\tau_{\mathcal{H}}, v_{\mathcal{H}}\right)$ intensity color plots of the spatiotemporal response of forward Bragg diffraction (FBD) and Bragg diffraction (BD) in Laue-case geometry shown in Figs. 8 and 9, which are analogs of the Bragg-case shown in Figs. 5 and 6.

Figure 8 shows 2D plots for the practically unbounded incident wave front. Unlike the Bragg case, in the Lauecase geometry the intensity fronts of the diffracted wave fields are always strongly inclined. This is a consequence of the nonvanishing angular dispersion in Laue geometry, in agreement with Eqs. (22) and (23).

Laue-case FBD is truly limited in time, both for observations made at a single point or over an extended field of view: the duration is always $\mathcal{T}_{d}$ (21), which can be changed by $d, \theta$, and $\eta$. The Laue-case BD is limited in time for an observer who measures the field at a single point, and its duration is the same as in FBD, namely, $\mathcal{T}_{d}$. However, for an observer that collects the reflected $\mathrm{x}$ rays over some region in space, the duration of $\mathrm{BD}$ depends on the extent of the field of view. For an infinite field of view the duration is infinite.

If the incident wave front is now strongly bounded, as assumed for calculations of the 2D plots presented in Fig. 9, then BD is limited to the region of the Bragg's law dispersion envelope. As a result, the duration of BD for an observer with an infinite field of view becomes limited to $\mathcal{T}_{d} / b$. We obtained this result also by ray tracing the wave fronts in Fig. 2, which represents the limiting case of an extremely bounded incident wave front.

From the above examples it is clear that there is no unambiguous answer to the question what is the duration of x-ray diffraction in Laue-case geometry. Depending on the conditions of the experiment, it can be either $\mathcal{T}_{d}$, or $\mathcal{T}_{d} / b$, or even arbitrarily long. The duration of $\mathrm{BD}$ in the Laue case can be varied not only by decreasing the crystal thickness, as was suggested in [15], but also by varying $\mathcal{T}_{d}$ or $\mathcal{T}_{d} / b$ through the asymmetry angle $\eta$ and asymmetry factor $b$, as follows from (21).

The lateral spread $v_{\mathcal{H}}$ of FBD and BD in Laue-case geometry appears to be limited to $v_{\mathcal{H}}^{(\max )}$ if the incident beam has a bounded wave front. This is a well-known result of the dynamical theory, supported by many experiments, reviewed in detail, e.g., in [11]. Figure 9 demonstrates how the limited-in-time crystal response correlates with the limited lateral spread. Using these graphs, one can find that the maximal lateral spread is given by

$$
\boldsymbol{v}_{0}^{(\max )}=d \sin 2 \theta / \gamma_{H}, \quad \boldsymbol{v}_{H}^{(\max )}=d \sin 2 \theta / \gamma_{0} .
$$

The same values can be obtained using ray tracing of the wave fronts in Fig. 2. In agreement with (64), we find from Fig. $2 A A^{\prime}=v_{0}^{(\max )}=d \sin 2 \theta / \gamma_{H}$, and $B B^{\prime}=v_{H}^{(\max )}=$ $d \sin 2 \theta / \gamma_{0}$.

\section{APPLICATIONS}

\section{A. Self-seeding of XFELs}

Understanding spatiotemporal dependencies in Bragg diffraction of $\mathrm{x}$ rays has immediate practical implications, in particular for self-seeding of $\mathrm{x}$-ray free-electron lasers (XFELs). The self-seeding scheme uses an upstream XFEL to generate an intense $\mathrm{X}$-ray pulse via self-amplified spontaneous emission (SASE). The relatively broad-bandwidth SASE pulse is then put through an x-ray monochromator to generate a monochromatic seed for the downstream XFEL undulators, which in turn amplifies the narrow bandwidth seed to produce fully coherent $x$ rays $[35,36]$. However, traditional two- or four-bounce monochromators induce a large delay ( $>10 \mathrm{ps}$ ) of the $\mathrm{x}$ rays, which in turn 
requires an impractically long $(\sim 40 \mathrm{~m})$ electron beam transfer line.

A very clever, readily realizable idea of a "wake" monochromator that produces a monochromatic x-ray seed at an optimal $\approx 20$ fs delay has been proposed by Geloni et al. [37,38], and recently realized at the Linac Coherent Light Source XFEL by an international team lead by Emma [39].

In the original proposal $[37,38]$ the authors applied the equations of the dynamical theory of $x$-ray diffraction in crystals to calculate numerically the time dependence and strength of the monochromatic seed propagating in forward direction. The action of the monochromator crystal in the Bragg-transmission geometry was interpreted in terms of a Bragg diffraction (BD) band-stop filter. The underlying physics is actually related to forward Bragg diffraction (FBD). We have discussed in detail its properties in the symmetric Bragg-case geometry relevant for self-seeding in [1]. We showed that, first, the characteristic time for FBD is $\mathcal{T}_{0}$ (55), substantially different (shorter) than the characteristic BD time $\mathcal{T}_{\Lambda}$ (43), and therefore the crystal in FBD generates a seed with a broader spectrum than a BD band-stop filter would do. Second, it was shown that the intensity of the monochromatic seed is $\propto 1 / \mathcal{T}_{0}^{2}$, cf. Eq. (56), which can therefore be enhanced by varying parameters composing $\mathcal{T}_{0}$. Similarly, its time delay $t_{s}=$ $26 \mathcal{T}_{0}$ (see Fig. 4), and its duration $\Delta t_{s}=16.5 \mathcal{T}_{0}$ can be tailored by changing $\mathcal{T}_{0}$, which can be done practically by adjusting the extinction length $\bar{\Lambda}_{H}^{\text {(s) }}$ (for example, by choosing another reflection or asymmetry parameter), or by changing the crystal thickness. A limitation of this scheme has been also identified in [1]. It is due to the lateral shift of the FBD signal. This is a very generic effect, caused by the Bragg's law dispersion, as discussed in Sec. IIC of the present paper.

The theory developed in the present paper allows us to diversify the variety of possible forward diffraction selfseeding monochromator schemes. First of all, forward Bragg diffraction in Laue-case geometry is a competitive approach. The possibility of applying FBD in Laue-case geometry for self-seeding becomes immediately apparent from the derived equivalence of the forward diffraction plane-wave response functions $\left|\tilde{G}_{00}\left(\tau_{0}\right)\right|^{2}$ in the Laue-case geometry (62), and of its counterpart (56) in the Bragg-case geometry. The equivalence holds for small $\tau_{0} \ll \mathcal{T}_{d}$, which is the range most appropriate for self-seeding of femtosecond long XFEL pulses. As has been established in this paper, the time constant of forward Bragg diffraction $\mathcal{T}_{0}(55)$ is common for all symmetric or asymmetric, transmission or reflection scattering geometries, and is the only parameter which defines the strength, delay, and duration of FBD and therefore of the monochromatic seed. These properties advance FBD both in Bragg and Lauecase geometries, including asymmetric ones, to a universal approach for the generation of monochromatic, delayed seeds for self-seeded XFELs. The physics is controlled by the parameters which compose $\mathcal{T}_{0}$ (55): the magnitude of the effective crystal thickness $d / \gamma_{0}$, and the extinction length $\bar{\Lambda}_{H}^{(\mathrm{s})}$ in the symmetric Bragg reflection. Table I in Appendix E provides some useful data for Bragg reflections in diamond, which can be used to select the Bragg reflection most appropriate for the desired application. Similar data for silicon and $\mathrm{Al}_{2} \mathrm{O}_{3}$ crystals can be found in [18].

There is no universal answer to the question: which geometry is better, Bragg or Laue? We investigate certain aspects of this question below, where for simplicity we have restricted our analysis to the symmetric diffraction geometries, defined by $\eta=0$ in the Bragg case-Fig. 1(a), and by $\eta=\pi / 2$ in the Laue case-Fig. 1(b).

If the experimenter highly values operating the selfseeding monochromator over as large a spectral tuning range as is possible, then the Laue-case geometry may be a better choice. The strongest variation of the photon energy $E$ with the glancing angle of incidence to the reflecting atomic planes $\theta$ takes place for small $\theta \leqslant \pi / 6$, i.e., in the linear range of Bragg's law $E \sin \theta=E_{H}$ (3). We write here Bragg's law in terms of photon energy $E$ and Bragg energy $E_{H}=H c \hbar / 2$, the smallest photon energy for which Bragg's law can be fulfilled (at $\theta=\pi / 2$ ). In the symmetric Laue-case geometry the effective thickness $d / \gamma_{0}=$ $d / \cos \theta$ does not vary much if $\theta$ is small, unlike the Bragg case in which $d / \gamma_{0}=d / \sin \theta$. Therefore, a large variation in $E$ is accompanied in the Laue-case geometry with a small variation in $d / \gamma_{0}$ and therefore in $\mathcal{T}_{0}$, resulting in a rather stable seed power and time delay of the seed over a large range of photon energies. In the Bragg-case geometry this is not the case. In addition, the Laue-case geometry at small $\theta$ allows for using thicker crystals for the same $\mathcal{T}_{0}$ as compared to the similar situation in the Bragg case. This may represent a technical advantage since the fabrication of thin crystals is typically more challenging.

Using small $\theta$ angles, however, also has its disadvantages. The lateral spatial shift, given by the Bragg's law dispersion envelope $\Pi^{2}\left(v_{0}-\tau_{0} c \cot \theta\right)$-Eq. (37), is $v_{0}=\tau_{0} c \cot \theta$, i.e., proportional to $\cot \theta$, and is maximal in the range of small $\theta$. This may not be significant for very short $\mathrm{x}$-ray pulses that can use short delay times $t_{s}$. However, if one wants to seed long XFEL pulses $\simeq 50-100 \mathrm{fs}$, then the Bragg-case scattering geometry close to backscattering $\theta \rightarrow \pi / 2$ would be a more advantageous option, albeit at a decrease in the spectral tuning range.

For example, the (733) Bragg reflection in diamond, having $\bar{\Lambda}_{H}^{(\mathrm{s})}=19.5 \mu \mathrm{m}$ (see Table I), can be used for self-seeding of $\simeq 100$ fs long pulses at the photon energy $E=14.4125 \mathrm{keV}$ of the nuclear resonance in ${ }^{57} \mathrm{Fe}$. In this case, the angle of incidence is near normal, with $\theta=$ $80.7^{\circ}$. Assuming a $d=200 \mu \mathrm{m}$ thick diamond crystal plate with surfaces perpendicular to (001), we obtain $\eta=$ $68.5^{\circ}, \mathcal{T}_{0}=6.5 \mathrm{fs}$, and that the first trailing maximum of 
FBD (the seed) appears at $t_{s}=26 \mathcal{T}_{0}=168 \mathrm{fs}$ with a duration of $\Delta t_{s}=107 \mathrm{fs}$. The lateral shift is only $v_{0} \simeq$ $10 \mu \mathrm{rad}$ after $200 \mathrm{fs}$ in this case, while the spectral bandwidth of the FBD seed is $\Delta E_{0} \simeq \Delta E_{H}(\mathcal{A} / 2 \pi) \simeq 30 \mathrm{meV}$.

\section{B. Ultrafast time measurements by mapping time on space}

Angular dispersion in asymmetric Bragg diffraction results in an inclined intensity front of the diffracted wave fields. This effect is illustrated in Figs. 5 and 8, in Braggcase and Laue-case geometries, respectively. Inclination of the intensity front in asymmetric x-ray diffraction geometry was, to our knowledge, first explicitly derived using ray tracing in [40], where it was proposed to be used for x-ray pulse compression.

Here, we suggest using the effect of intensity front inclination for time measurements of ultrafast processes. The schematic drawing in Fig. 10 explains the idea. The inclined intensity front allows mapping time onto space, as different parts of the inclined front traverse and thus probe the sample at different times.

We assume that the process under study is triggered in the sample homogeneously over its extent by, for example, an external laser. If we then probe the sample with an inclined x-ray intensity front from an asymmetric crystal, then different transverse positions will be probed at different times, so that time dynamics can be extracted with a spatially resolved $\mathrm{x}$-ray detector. We assume that the $\mathrm{x}$-ray wave front is sufficiently broad so that we may ignore any small additional spatial shifts that take place due to Bragg's law dispersion.

To estimate the achievable time resolution, we first neglect the finite duration of the incident $\mathrm{x}$-ray pulse, the finite duration of Bragg diffraction, and the sample thickness. In this case, the resolution of the time measurements $\Delta t=(\Delta x / c) / \tan \beta=\Delta x /(c D)$ is determined by the spatial resolution of the detector $\Delta x$ and normalized

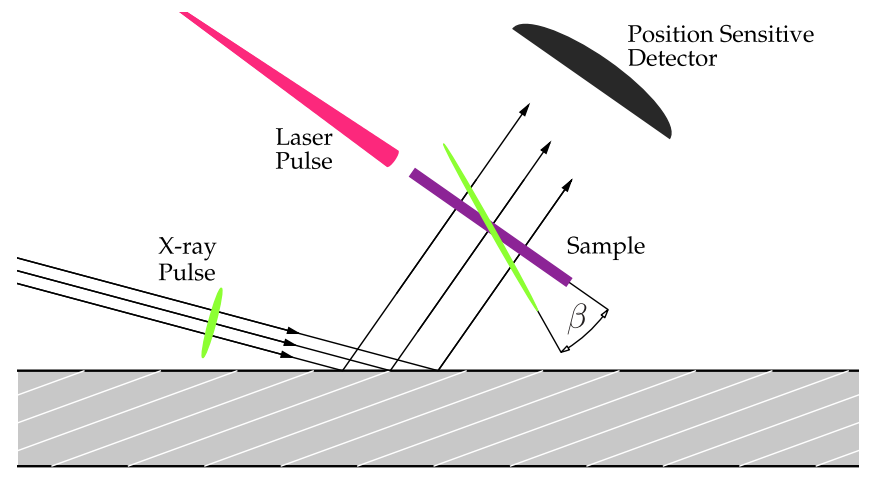

FIG. 10. Schematic of ultrafast time measurements by mapping time delay on space in asymmetric x-ray Bragg diffraction from a crystal. The intensity front is rotated by an angle $\beta$ upon asymmetric Bragg reflection of $\mathrm{x}$ rays. Here $\tan \beta=D$ is determined by normalized dispersion rate $D(22)$. dispersion rate $D$ (16). With $\Delta x \simeq 1-10 \mu \mathrm{m}$, and $D \simeq 1$, an estimate for the time resolution is $\Delta t \simeq 3-30 \mathrm{fs}$. The duration of the incident $\mathrm{x}$-ray pulse, the duration of Bragg diffraction, and the sample thickness will increase this number. The duration of the Bragg diffraction is $\mathcal{T}_{\Lambda}$ in Bragg-case or $\mathcal{T}_{d}$ in Laue-case geometry. By an appropriate choice of $\bar{\Lambda}_{H}^{\text {(s) }}$ and asymmetry factor $b$, the characteristic time of Bragg diffraction (42) can be made, however, as small as $\mathcal{T}_{\Lambda} \simeq 1 \mathrm{fs}$, i.e., smaller than the $\Delta t$. Tilting the sample to the $\mathrm{x}$-rays propagation direction (without tilting the intensity front) may result in a similar effect. However, tilting the intensity front in many cases may be advantageous, as it is decoupled from the propagation direction in the sample, which may be an important parameter of experiments, of diffraction experiments in particular.

\section{CONCLUSIONS}

The spatiotemporal response of crystals in x-ray Bragg diffraction resulting from excitation by an ultrashort, laterally confined $x$-ray pulse was studied theoretically. The theory developed in the paper presents an extension of the analysis in symmetric reflection geometry [1] to a generic case, which includes Bragg diffraction both in reflection (Bragg) and transmission (Laue) asymmetric scattering geometries.

The spatiotemporal response is presented as a product of a crystal-intrinsic plane-wave spatiotemporal response function and an envelope function defined by the crystalindependent transverse profile of the incident beam and the scattering geometry. The diffracted wave fields exhibit amplitude (or intensity) modulation perpendicular to the propagation direction due to two effects: angular dispersion and dispersion due to Bragg's law. Angular dispersion results in the inclination of the intensity front of Bragg diffraction in asymmetric geometries. Bragg's law dispersion produces a lateral spatial shift $v_{0}$ of photons emerging from the crystal with respect to the incident $\mathrm{X}$-ray pulse that increases linearly with time delay $\tau_{0}$. A simple general relationship $c \tau_{0}=v_{0} \tan \theta$ holds in all diffraction geometries. This effect was also interpreted in terms of the energy flow of the wave fields in the crystal.

The spatiotemporal plane-wave response functions in Bragg diffraction can be expressed in terms of three characteristic space and related to them three time parameters: (i) crystal thickness $d$ and $\mathcal{T}_{d}$ (21), (ii) extinction length $\bar{\Lambda}_{H}$ (40) and $\mathcal{T}_{\Lambda}$ (42), (iii) rescattering length $\bar{\Lambda}_{H}^{2} / d$ and $\mathcal{T}_{0}$ (55). The glancing angle of incidence $\theta$ and the asymmetry angle $\eta$ also enter the three time parameters, and, therefore, are factors that change the spatiotemporal response scale.

We address some practical applications of the developed theory. We show that forward Bragg diffraction (FBD) of $x$ rays in Laue-case geometry can be used for self-seeding of hard x-ray free-electron lasers, along with FBD in the Bragg-case geometry. Laue-case FBD is advantageous if 
a large spectral tuning range is required. We discuss also a possibility of using asymmetric diffraction for ultrafast time measurements with femtosecond resolution.

\section{ACKNOWLEDGMENTS}

The authors would like to thank Sasha Zholents for reading selected sections of the manuscript and valuable suggestions. This work is supported by the U.S. Department of Energy, Basic Energy Sciences, Office of Science, under Contract No. DE-AC02-06CH11357.

\section{APPENDIX A: RESPONSE FUNCTION IN REFLECTION (BRAGG) GEOMETRY}

To compute the temporal response of the forward diffracted wave, we must evaluate

$$
\tilde{G}_{00}\left(\xi_{0}\right)=-\frac{\mathcal{C}}{\mathcal{T}_{\Lambda}} \mathrm{e}^{-\mathrm{i} w_{H} \omega \xi_{0}} \int_{-\infty}^{\infty} \frac{\mathrm{d} y}{2 \pi} \mathrm{e}^{-\mathrm{i}\left(\xi_{0} / \mathcal{T}_{\Lambda}\right) y} \tilde{R}_{00}(y),
$$

where the $\tilde{R}_{00}(y)$ is given by subtracting $\mathcal{C}$ from (49). Since causality requires $\tilde{G}_{00}\left(\xi_{0}<0\right)=0$, we have found that the most convenient way to treat this particular problem is as an inverse Laplace transform. In the table of inverse Laplace transforms given by Erdélyi, Magnus, Oberhettinger, and Tricomi [41], we find that

$$
\begin{aligned}
\int_{0}^{\infty} d t e^{-p t} f(t) & =1-e^{-b\left(\sqrt{p^{2}+a^{2}}-p\right)} \\
\Rightarrow f(t) & =\frac{a b}{\sqrt{t(t+2 b)}} J_{1}[a \sqrt{t(t+2 b)}] .
\end{aligned}
$$

Now, we make the replacements: $t=\xi_{0}, p=-\mathrm{i} y / \mathcal{T}_{\Lambda}$, $a=1 / \mathcal{T}_{\Lambda}$, and $b=\mathcal{A} \mathcal{T}_{\Lambda} / 2$; then, (A2) is proportional to the approximate Bragg transmission. Thus, we have that

$$
\begin{aligned}
\int_{0}^{\infty} d \xi_{0} e^{\mathrm{i} y \xi_{0} / \mathcal{T}_{\Lambda}} f\left(\xi_{0}\right) & =e^{i \mathcal{A} / 2\left[ \pm \sqrt{y^{2}-1}-y\right]}-1 \\
\Rightarrow f\left(\xi_{0}\right) & =-\frac{\mathcal{A}}{2} \frac{J_{1}\left[\sqrt{\xi_{0}\left(\mathcal{A} \mathcal{T}_{\Lambda}+\xi_{0}\right)} / \mathcal{T}_{\Lambda}\right]}{\sqrt{\xi_{0}\left(\mathcal{A} \mathcal{T}_{\Lambda}+\xi_{0}\right)}} .
\end{aligned}
$$

In terms of the forward Bragg diffraction amplitude, (A3) implies that

$$
\begin{aligned}
\tilde{G}_{00}\left(\xi_{0}\right) & =-\frac{\mathcal{C}}{\mathcal{T}_{\Lambda}} \mathrm{e}^{-\mathrm{i} w_{H} \omega \xi_{0}} \int_{-\infty}^{\infty} \frac{\mathrm{d} y}{2 \pi} \mathrm{e}^{-\mathrm{i}\left(\xi_{0} / \mathcal{T}_{\Lambda}\right) y} \tilde{R}_{00}(y) \\
& =-\frac{\mathcal{C}}{2 \mathcal{T}_{0}} \frac{J_{1}\left[\sqrt{\frac{\xi_{0}}{\mathcal{T}_{0}}\left(1+\frac{\xi_{0}}{\mathcal{T}_{d}}\right)}\right]}{\sqrt{\frac{\xi_{0}}{\mathcal{T}_{0}}\left(1+\frac{\xi_{0}}{\mathcal{T}_{d}}\right)}} \mathrm{e}^{-\mathrm{i} w_{H} \omega \xi_{0}},
\end{aligned}
$$

with $\mathcal{T}_{0}=\mathcal{T}_{\Lambda} / \mathcal{A} \equiv 2 \gamma_{0}\left[\bar{\Lambda}_{H}^{(s)}\right]^{2} /(c d)$. On the other hand, the reflected wave is given by

$$
G_{0 H}\left(\xi_{H}\right)=\frac{G}{\mathcal{T}_{\Lambda}} \mathrm{e}^{-\mathrm{i} w_{H} \omega \xi_{H}} \int_{-\infty}^{\infty} \frac{d y}{2 \pi} \mathrm{e}^{-\mathrm{i}\left(\xi_{H} / \mathcal{T}_{\Lambda}\right) y} R_{0 H}(y),
$$

and the integrals are simple enough for MATHEMATICA to do; we find that

$$
\begin{aligned}
& \int_{1}^{\infty} \frac{d y}{2 \pi} \mathrm{e}^{-\mathrm{i}\left(\xi_{H} / \mathcal{T}_{\Lambda}\right) y}\left(-y+\sqrt{y^{2}-1}\right) \\
& \quad+\int_{-\infty}^{-1} \frac{d y}{2 \pi} \mathrm{e}^{-\mathrm{i}\left(\xi_{H} / \mathcal{T}_{\Lambda}\right) y}\left(-y-\sqrt{y^{2}-1}\right) \\
& =2 \mathrm{i} \int_{1}^{\infty} \frac{d y}{2 \pi} \sin \left(\xi_{H} y / \mathcal{T}_{\Lambda}\right)\left(y-\sqrt{y^{2}-1}\right) \\
& =\mathrm{i} \frac{J_{1}\left(\xi_{H} / \mathcal{T}_{\Lambda}\right)}{2 \xi_{H} / \mathcal{T}_{\Lambda}} \operatorname{sgn}\left(\xi_{H} / \mathcal{T}_{\Lambda}\right) \\
& +\mathrm{i} \frac{\left(\xi_{H} / \mathcal{T}_{\Lambda}\right) \cos \left(\xi_{H} / \mathcal{T}_{\Lambda}\right)-\sin \left(\xi_{H} / \mathcal{T}_{\Lambda}\right)}{\pi\left(\xi_{H} / \mathcal{T}_{\Lambda}\right)^{2}}
\end{aligned}
$$

and

$$
\begin{aligned}
& \int_{-1}^{1} \frac{d y}{2 \pi}\left[\mathrm{i} y \sin \left(\xi_{H} y / \mathcal{T}_{\Lambda}\right)+\mathrm{i} \sqrt{1-y^{2}} \cos \left(\xi_{H} y / \mathcal{T}_{\Lambda}\right)\right] \\
& =\mathrm{i} \frac{J_{1}\left(\xi_{H} / \mathcal{T}_{\Lambda}\right)}{2 \xi_{H} / \mathcal{T}_{\Lambda}} \\
& -\mathrm{i} \frac{\left(\xi_{H} / \mathcal{T}_{\Lambda}\right) \cos \left(\xi_{H} / \mathcal{T}_{\Lambda}\right)-\sin \left(\xi_{H} / \mathcal{T}_{\Lambda}\right)}{\pi\left(\xi_{H} / \mathcal{T}_{\Lambda}\right)^{2}} .
\end{aligned}
$$

Adding these two, we obtain

$$
\begin{aligned}
G_{0 H}\left(\xi_{H}\right) & =\frac{G}{\mathcal{T}_{\Lambda}} \mathrm{e}^{-\mathrm{i} w_{H} \omega \xi_{H}} \int_{-\infty}^{\infty} \frac{d y}{2 \pi} \mathrm{e}^{-\mathrm{i}\left(\xi_{H} / \mathcal{T}_{\Lambda}\right) y} R_{0 H}(y) \\
& =\mathrm{i} \frac{G}{\mathcal{T}_{\Lambda}} \mathrm{e}^{-\mathrm{i} w_{H} \omega \xi_{H}} \frac{J_{1}\left(\xi_{H} / \mathcal{T}_{\Lambda}\right)}{\xi_{H} / \mathcal{T}_{\Lambda}} \Theta\left(\xi_{H} / \mathcal{T}_{\Lambda}\right) .
\end{aligned}
$$

\section{APPENDIX B: RESPONSE FUNCTION IN TRANSMISSION (LAUE) GEOMETRY}

To calculate response functions in Laue-case diffraction geometry we use Eqs. (18), (58), (59), and (41) in the form $\Omega=-y / \mathcal{T}_{\Lambda}+w_{H} \omega$, as $b>1$ in Laue-case geometry. As a result we obtain

$$
\begin{aligned}
G_{00}\left(\xi_{0}\right) & =-\frac{\mathcal{C}}{\mathcal{T}_{\Lambda}} \mathrm{e}^{-\mathrm{i} w_{H} \omega \xi_{0}} I_{0}, \\
I_{0} & =\int_{-\infty}^{\infty} \frac{d y}{2 \pi} \mathrm{e}^{-\mathrm{i} \xi_{0} y} W(y), \quad \zeta_{0}=-\frac{\xi_{0}}{\mathcal{T}_{\Lambda}}+\frac{\mathcal{A}}{2}, \\
W(y) & =\cos \left(\frac{\mathcal{A}}{2} \sqrt{y^{2}+1}\right)+\mathrm{i} y \frac{\sin \left(\frac{\mathcal{A}}{2} \sqrt{y^{2}+1}\right)}{\sqrt{y^{2}+1}},
\end{aligned}
$$

and 


$$
\begin{aligned}
G_{0 H}\left(\xi_{H}\right) & =-\mathrm{i} \frac{\mathcal{C} G}{\mathcal{T}_{\Lambda}} \mathrm{e}^{-\mathrm{i} w_{H} \omega \xi_{H}} I_{H}, \\
I_{H} & =\int_{-\infty}^{\infty} \frac{d y}{2 \pi} \mathrm{e}^{-\mathrm{i} \xi_{H} y} V(y), \quad \zeta_{H}=-\frac{\xi_{H}}{\mathcal{T}_{\Lambda}}+\frac{\mathcal{A}}{2}, \\
V(y) & =\frac{\sin \left(\frac{\mathcal{A}}{2} \sqrt{y^{2}+1}\right)}{\sqrt{y^{2}+1}} .
\end{aligned}
$$

The Fourier integral $I_{H}$ in (B2) is a tabulated integral [42] and can be calculated analytically, as was previously carried out in solving similar problems $[11,16,25]$ :

$$
I_{H}=\frac{1}{2} J_{0}\left(\sqrt{(\mathcal{A} / 2)^{2}-\zeta_{H}^{2}}\right) \Theta\left(\frac{\mathcal{A}}{2}+\zeta_{H}\right) \Theta\left(\frac{\mathcal{A}}{2}-\zeta_{H}\right) .
$$

Here $\Theta()$ is the Heaviside unit step function whose value is zero for negative argument and one for positive argument.

The Fourier integral $I_{0}$ in (B1) can be calculated using the property $I_{0}=\partial I_{H} / \partial(\mathcal{A} / 2)-\partial I_{H} / \partial \zeta[11,16,25]$ resulting in

$$
\begin{aligned}
I_{0}= & \tilde{I}_{0}+2 \delta\left(\frac{\mathcal{A}}{2}-\zeta_{0}\right), \\
\tilde{I}_{0}= & -\frac{\mathcal{A} / 2+\zeta_{0}}{2 \sqrt{(\mathcal{A} / 2)^{2}-\zeta_{0}^{2}}} \\
& \times J_{1}\left(\sqrt{(\mathcal{A} / 2)^{2}-\zeta_{0}^{2}}\right) \Theta\left(\frac{\mathcal{A}}{2}+\zeta_{0}\right) \Theta\left(\frac{\mathcal{A}}{2}-\zeta_{0}\right) .
\end{aligned}
$$

Here the $\delta$ function appears as a result of differentiating the step functions. Finally with (B3) and (B4) and definition of $\zeta_{0}$ and $\zeta_{H}$ in (B1) and (B2) we arrive at the following analytical expressions for the plane-wave response functions in Laue-case geometry:

$$
\begin{aligned}
G_{00}\left(\xi_{0}\right)= & \tilde{G}_{00}\left(\xi_{0}\right)+\mathcal{C} \delta\left(\xi_{0}\right), \\
\tilde{G}_{00}\left(\xi_{0}\right)= & \frac{\mathcal{C}}{2 \mathcal{T}_{0}} \mathrm{e}^{-\mathrm{i} w_{H} \omega \xi_{0}}\left(1-\frac{\xi_{0}}{\mathcal{T}_{d}}\right) \\
& \times \frac{J_{1}\left[\sqrt{\frac{\xi_{0}}{\mathcal{T}_{0}}\left(1-\frac{\xi_{0}}{\mathcal{T}_{d}}\right)}\right]}{\sqrt{\frac{\xi_{0}}{\mathcal{T}_{0}}\left(1-\frac{\xi_{0}}{\mathcal{T}_{d}}\right)}} \Theta\left(\xi_{0}\right) \Theta\left(\mathcal{T}_{d}-\xi_{0}\right), \quad(\mathrm{B} 5) \\
G_{0 H}\left(\xi_{H}\right)= & -\mathrm{i} \frac{\mathcal{C G}}{2 \mathcal{T}_{\Lambda}} \mathrm{e}^{-\mathrm{i} w_{H} \omega \xi_{H}} \\
& \times J_{0}\left[\sqrt{\frac{\xi_{H}}{\mathcal{T}_{0}}\left(1-\frac{\xi_{H}}{\mathcal{T}_{d}}\right)}\right] \Theta\left(\xi_{H}\right) \Theta\left(\mathcal{T}_{d}-\xi_{H}\right) .
\end{aligned}
$$

With one exception, these expressions agree with relevant expressions obtained by Malgrange and Graeff in
[16], where diffraction of short x-ray pulses with infinite wave front in the asymmetric Laue case was studied analytically. Unlike the expression for forward diffraction presented in [16], Eq. (B5) contains the delta function, which represents the prompt response in the forward diffraction due to spectral components far from the Bragg diffraction region that propagate essentially diffractionfree through the crystal.

We note also that a reference system $\left(x^{\prime}, z^{\prime}\right)$ was used in [16] attached to the crystal rear surface. Unlike this, we are using in our treatment for each diffracted wave field its own reference system $\left(\hat{\boldsymbol{u}}_{\mathcal{H}}, \hat{\boldsymbol{v}}_{\mathcal{H}}\right)$. We are also using a different approach to calculate the vacuum wave vector of the diffracted wave (7)-(10). Because of these differences, the expressions for the spatiotemporal variables $\xi_{\mathcal{H}}$ (20)-(23) similar variables in [16] —Eq. (28) — may appear at a first glance to be very different. However, our detailed comparison shows that they are actually identical. So mathematically our results and results of paper [16] for the delayed parts of the response functions are in agreement, except for the delta function in Eq. (B5).

\section{APPENDIX C: MAPPING TIME ON LATERAL SPACE SHIFT}

The relationship $v_{\mathcal{H}}=\tau_{\mathcal{H}} c \cot \theta$ between the time delay $\tau_{\mathcal{H}}$ and the spatial shift $\boldsymbol{v}_{\mathcal{H}}$ in Bragg diffraction, representing the trace of the Bragg's law dispersion envelope $\Pi\left(v_{\mathcal{H}}-\tau_{\mathcal{H}} c \cot \theta\right)$ in Eq. (36), can be derived alternatively by combining Ewald's concept of the crystal wave field [4] with the concept of energy flow introduced by von Laue [43].

These concepts lead to the following picture of physical processes involved in X-ray Bragg diffraction in crystals. It is illustrated graphically in Figs. 11(a) and 11(b), schematically presenting diffraction in the Bragg-case geometry and in the Laue-case geometry, respectively.

An incident monochromatic plane wave with wave vector $\boldsymbol{K}_{0}$ excites monochromatic wave fields in the crystal given by Eq. (1), where each wave field is associated with a tie point on one of the brunches of the dispersion surface, which we number below by $\nu$. The energy flow for each wave field in a perfect crystal is given by the wave field Poynting vector [43], which is directed along the normal to the dispersion surface taken at the tie point of the surface representing the field [44]. The Poynting vector is parallel to the vector of group velocity $\boldsymbol{V}_{\nu}$. As a result, different monochromatic wave fields propagate, first, along different paths of different lengths, and second, with different group velocities $\boldsymbol{V}_{\nu}$. At the exit surface, the wave field breaks up into independent plane-wave fields, one with the wave vector $\boldsymbol{K}_{0}$ propagating in the direction $\hat{\boldsymbol{u}}_{0}$, and another with the wave vector $\boldsymbol{K}_{H}$ propagating in the direction $\hat{\boldsymbol{u}}_{H}$. One should note that the concept of the energy flow works with one substantial limitation: it is not applicable in the total reflection region in Bragg-case geometry, as there is 


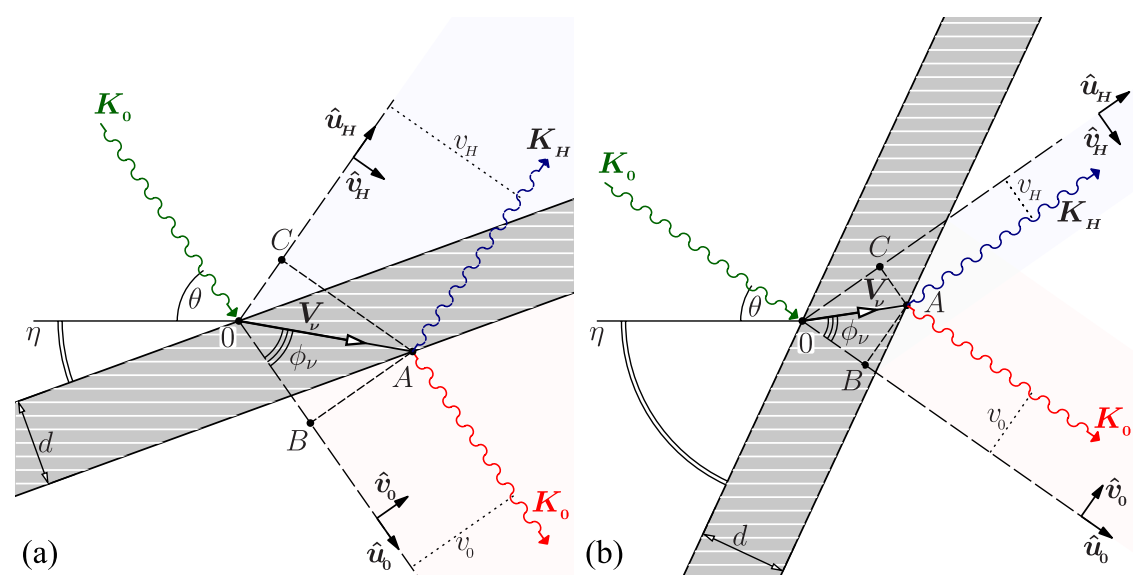

FIG. 11. Schematic presentation of two-beam x-ray Bragg diffraction from a crystal (a) in the reflection (Bragg) scattering geometry and (b) in the transmission (Laue) scattering geometry. The direction of the energy flow by the crystal wave field is indicated by the vector of the group velocity $\boldsymbol{V}_{\nu}$, at an angle $\phi_{\nu}$ to the direction of the incident wave.

no propagating through the crystal wave fields in this case. It is applicable, however, outside the total reflection region. Because of this, the wave with the wave vector $\boldsymbol{K}_{H}$ is shown in Fig. 11(a) propagating along the line starting at point $A$.

Using Figs. 11(a) and 11(b) we calculate for the spatial shifts $v_{\mathcal{H}}$ and delays $\tau_{\mathcal{H}}$ :

$$
\begin{gathered}
v_{0} \equiv A B=O A \sin \phi_{\nu}, \quad \tau_{0}=\frac{O A}{V_{\nu}}-\frac{O B}{c}, \\
v_{H} \equiv A C=O A \sin \left(2 \theta-\phi_{\nu}\right), \quad \tau_{H}=\frac{O A}{V_{\nu}}-\frac{O C}{c} .
\end{gathered}
$$

The magnitude of the group velocity can be given, as derived in Appendix D, by

$$
\frac{V_{\nu}}{c}=\frac{\cos \theta}{\cos \left(\theta-\phi_{\nu}\right)} \text {. }
$$

We use in Eq. $(\mathrm{C} 1)$ the fact that propagation along $O B$ $\left(\phi_{\nu}=0\right)$ or $O C\left(\phi_{\nu}=2 \theta\right)$ takes place with the speed of light in vacuum $c$, in agreement with Eq. (C3). Using the relationships $O B=O A \cos \phi_{\nu}, O C=O A \cos \left(2 \theta-\phi_{\nu}\right)$, and Eq. (C3), we obtain

$$
v_{\mathcal{H}}=\tau_{\mathcal{H}} c \cot \theta, \quad \mathcal{H}=(0, H) .
$$

This relationship is valid in the general case of asymmetric diffraction, both for Bragg and Laue scattering geometries. It maps temporal onto spatial scales in Bragg diffraction, in agreement with the Bragg's law dispersion envelope $\Pi\left(v_{\mathcal{H}}-\tau_{\mathcal{H}} c \cot \theta\right)$ in Eq. (36). Uncertainty relationships are always valid, and therefore Eq. (C4) is actually applied not for absolutely monochromatic waves, or waves localized in time and space, but rather for wave packets with certain spectral and momentum distributions.

\section{APPENDIX D: WAVE FIELD GROUP VELOCITY IN THE CRYSTAL VS PROPAGATION ANGLE}

The group velocity vector $\boldsymbol{V}_{\nu}$ is given by $[11,43,44]$

$$
\boldsymbol{V}_{\nu}=c \frac{\hat{\boldsymbol{u}}_{0}+\hat{\boldsymbol{u}}_{H} R_{\nu}^{2}}{1+R_{\nu}^{2}}
$$

where $R_{\nu}$ is defined in Eq. (39), and $\nu$ numbers brunches of the dispersion surface.

The absolute value of the group velocity $V_{\nu}$ can be calculated by taking the magnitude of Eq. (D1) and recalling that $\hat{\boldsymbol{u}}_{0} \hat{\boldsymbol{u}}_{H}=\cos 2 \theta$ :

$$
V_{\nu}=c \frac{\sqrt{1+2 R_{\nu}^{2} \cos 2 \theta+R_{\nu}^{4}}}{1+R_{\nu}^{2}} .
$$

The angle $\phi_{\nu}$ between the direction of the group velocity vector $\boldsymbol{V}_{\nu}$ and optical axis $\hat{\boldsymbol{u}}_{0}$ is determined from the vector scalar product $\cos \phi_{\nu}=\hat{\boldsymbol{u}}_{0} \boldsymbol{V}_{\nu} / V_{\nu}$, which using Eq. (D1) becomes

$$
\cos \phi_{\nu}=\frac{1+R_{\nu}^{2} \cos 2 \theta}{\sqrt{1+2 R_{\nu}^{2} \cos 2 \theta+R_{\nu}^{4}}} .
$$

Combining (D2) and (D3) we obtain the following relationship between the magnitude of wave field group velocity $V_{\nu}$ and its direction $\phi_{\nu}$ :

$$
\frac{V_{\nu}}{c}=\frac{\cos \theta}{\cos (\theta-\phi)}
$$

Equation (D4) gives a physically reasonable result. The direction of the wave field propagation under the Bragg diffraction condition is at $\phi=\theta$, in which case the group velocity $V_{\nu}=c \cos \theta$ is less than speed of light in vacuum. Far from Bragg diffraction conditions $\phi=0$ or $\phi=2 \theta$, resulting in a reasonable solution $V_{\nu}=c$. 


\section{APPENDIX E: BRAGG REFLECTIONS IN DIAMOND}

TABLE I. Allowed Bragg reflections $\boldsymbol{H}=(h k l)$ in diamond crystals and their parameters relevant to the present studies: Bragg energy $E_{H}=h c / 2 d_{H}$, the extinction length $\bar{\Lambda}_{H}^{(s)}(40)$, the Bragg's law correction $w_{H}^{(s)}(44)$, the energy width $\Delta E_{H}$ (at $\left.\theta=\pi / 2\right)$. These parameters are calculated using interplanar distance $d_{H}=3.56712(2) \AA$ in diamond crystals at $T=298 \mathrm{~K}$ $[28,29]$, a Debye temperature of $2230 \mathrm{~T}$ [30], anomalous scattering factors from [31,32], and assuming $|P|=1$, i.e., for the $\sigma$-polarization component. If the $\pi$ component is involved, the tabulated $\bar{\Lambda}_{H}^{(s)}$ values should be increased by $1 /|P|=\left|\cos ^{-1} 2 \theta\right|$, cf. Eq. (40).

\begin{tabular}{|c|c|c|c|c|c|c|}
\hline$h$ & $\begin{array}{r}\boldsymbol{H} \\
k\end{array}$ & $l$ & $\begin{array}{c}E_{H} \\
{[\mathrm{keV}]}\end{array}$ & $\begin{array}{c}\bar{\Lambda}_{H}^{(s)} \\
{[\mu \mathrm{m}]}\end{array}$ & $\begin{array}{c}w_{H}^{(s)} \\
\times 10^{-5}\end{array}$ & $\begin{array}{c}\Delta E_{H} \\
{[\mathrm{meV}]}\end{array}$ \\
\hline 1 & 1 & 1 & 3.01034 & 1.09 & 8.17 & 192.0 \\
\hline 2 & 2 & 0 & 4.91561 & 1.98 & 3.04 & 106.0 \\
\hline 3 & 1 & 1 & 5.76401 & 3.74 & 2.20 & 56.0 \\
\hline 4 & 0 & 0 & 6.95161 & 3.63 & 1.51 & 60.6 \\
\hline 3 & 3 & 1 & 7.57532 & 5.89 & 1.27 & 35.8 \\
\hline 4 & 2 & 2 & 8.51391 & 5.03 & 1.00 & 44.5 \\
\hline 3 & 3 & 3 & 9.03035 & 7.83 & 0.89 & 27.3 \\
\hline 5 & 1 & 1 & 9.03035 & 7.83 & 0.89 & 27.3 \\
\hline 4 & 4 & 0 & 9.83108 & 6.41 & 0.75 & 35.9 \\
\hline 5 & 3 & 1 & 10.2815 & 9.82 & 0.69 & 22.6 \\
\hline 6 & 2 & 0 & 10.9914 & 7.87 & 0.60 & 29.2 \\
\hline 5 & 3 & 3 & 11.3961 & 11.9 & 0.56 & 19.1 \\
\hline 4 & 4 & 4 & 12.0404 & 9.44 & 0.50 & 25.0 \\
\hline 5 & 1 & 5 & 12.4110 & 14.2 & 0.47 & 16.4 \\
\hline 7 & 1 & 1 & 12.4110 & 14.2 & 0.47 & 16.4 \\
\hline 6 & 4 & 2 & 13.0051 & 11.1 & 0.43 & 21.3 \\
\hline 5 & 5 & 3 & 13.3489 & 16.7 & 0.40 & 14.3 \\
\hline 7 & 3 & 1 & 13.3489 & 16.7 & 0.40 & 14.3 \\
\hline 8 & 0 & 0 & 13.9030 & 13.0 & 0.37 & 18.6 \\
\hline 7 & 3 & 3 & 14.2251 & 19.5 & 0.36 & 12.6 \\
\hline 6 & 6 & 0 & 14.7464 & 15.1 & 0.33 & 16.5 \\
\hline 8 & 2 & 2 & 14.7464 & 15.1 & 0.335 & 16.5 \\
\hline 7 & 5 & 1 & 15.0504 & 22.5 & 0.322 & 10.8 \\
\hline 8 & 4 & 0 & 15.5440 & 17.3 & 0.301 & 14.7 \\
\hline 7 & 5 & 3 & 15.8328 & 25.7 & 0.291 & 9.9 \\
\hline 9 & 1 & 1 & 15.8328 & 25.7 & 0.291 & 9.9 \\
\hline 6 & 6 & 4 & 16.3027 & 19.7 & 0.274 & 12.9 \\
\hline 9 & 3 & 1 & 16.5783 & 29.2 & 0.265 & 8.5 \\
\hline 8 & 4 & 4 & 17.0276 & 22.3 & 0.251 & 11.6 \\
\hline 7 & 5 & 5 & 17.2916 & 33.0 & 0.243 & 7.8 \\
\hline 7 & 7 & 1 & 17.2916 & 33.0 & 0.243 & 7.8 \\
\hline 9 & 3 & 3 & 17.2916 & 33.0 & 0.243 & 7.8 \\
\hline 10 & 0 & 2 & 17.7229 & 25.1 & 0.232 & 10.1 \\
\hline 8 & 6 & 2 & 17.7229 & 25.1 & 0.232 & 10.1 \\
\hline 7 & 7 & 3 & 17.9767 & 37.2 & 0.225 & 6.9 \\
\hline 9 & 5 & 1 & 17.9767 & 37.2 & 0.225 & 6.9 \\
\hline 9 & 5 & 3 & 18.6366 & 41.6 & 0.210 & 6.1 \\
\hline 10 & 4 & 2 & 19.0375 & 31.5 & 0.201 & 8.1 \\
\hline 11 & 1 & 1 & 19.2740 & 46.4 & 0.196 & 5.4 \\
\hline 7 & 7 & 5 & 19.2740 & 46.4 & 0.196 & 5.4 \\
\hline 8 & 8 & 0 & 19.6618 & 35.1 & 0.188 & 7.2 \\
\hline 11 & 3 & 1 & 19.8909 & 51.6 & 0.184 & 5.0 \\
\hline 9 & 5 & 5 & 19.8909 & 51.6 & 0.184 & 5.0 \\
\hline 9 & 7 & 1 & 19.8909 & 51.6 & 0.184 & 5.0 \\
\hline
\end{tabular}


[1] R. R. Lindberg and Y. V. Shvyd'ko, Phys. Rev. ST Accel. Beams 15, 050706 (2012).

[2] F. N. Chukhovskii and E. Förster, Acta Crystallogr. Sect. A 51, 668 (1995).

[3] J. S. Wark and R. W. Lee, J. Appl. Crystallogr. 32, 692 (1999).

[4] P. P. Ewald, Ann. Phys. (Leipzig) 54, 519 (1917).

[5] M. von Laue, Exakt. Naturwiss. 10, 133 (1931).

[6] W.H. Zachariasen, Theory of X-Ray Diffraction in Crystals (John Wiley \& Sons, Inc., New York, 1945), reprinted by Dover Publications, New York, 1967.

[7] M. von Laue, Röntgenstrahl-Interferenzen (Akademische Verlagsgesellschaft, Frankfurt am Main, 1960).

[8] B. W. Batterman and H. Cole, Rev. Mod. Phys. 36, 681 (1964).

[9] Z. G. Pinsker, Dynamical Scattering of X rays in Crystals (Springer, Berlin, 1978).

[10] Z.G. Pinsker, Rentgenovskaya Kristallooptika (Dynamical Scattering of $X$ rays in Crystals) (Nauka, Moscow, 1982).

[11] A. Authier, Dynamical Theory of X-Ray Diffraction, IUCr Monographs on Crystallography (Oxford University Press, Oxford, New York, 2001), Vol. 11.

[12] S. D. Shastri, P. Zambianchi, and D. M. Mills, Proc. SPIE Int. Soc. Opt. Eng. 4143, 69 (2001).

[13] S.D. Shastri, P. Zambianchi, and D. M. Mills, J. Synchrotron Radiat. 8, 1131 (2001).

[14] D. Siddons, in NSLS Activity Report 2001, Science Highlights (NSLS, Brookhaven, NY, 2001), pp. 2-125-2128.

[15] W. Graeff, J. Synchrotron Radiat. 9, 82 (2002).

[16] C. Malgrange and W. Graeff, J. Synchrotron Radiat. 10, 248 (2003).

[17] D. P. Siddons, AIP Conf. Proc. 705, 997 (2004).

[18] Y. Shvyd'ko, X-Ray Optics-High-Energy-Resolution Applications, Optical Sciences (Springer, Berlin, 2004), Vol. 98.

[19] W. Graeff, J. Synchrotron Radiat. 11, 261 (2004).

[20] V. A. Bushuev, J. Synchrotron Radiat. 15, 495 (2008).

[21] The electric field inside the crystal is practically transverse, as a result it can be presented in one of the two orthogonal linear polarization states with polarization vectors normal to $\boldsymbol{K}_{0}$ or $\boldsymbol{K}_{0}+\boldsymbol{H}$, respectively. The $\sigma$-polarization components are usually defined to be parallel to $\left(\boldsymbol{K}_{0}+\boldsymbol{H}\right) \times \boldsymbol{K}_{0}$, i.e., perpendicular to the scattering plane, while $\pi$ components are defined to be lying in the scattering plane.

[22] Y. Kagan, A. M. Afanas'ev, and V. G. Kohn, J. Phys. C 12, 615 (1979).

[23] H. Wagner, Z. Phys. 146, 127 (1956).

[24] G. Geloni, V. Kocharyan, and E. Saldin, arXiv:1203.6442.
[25] N. Kato, Jpn. J. Appl. Phys. 39, 2225 (1968).

[26] The definition of the extinction length varies by a factor of $2 \pi$ in the dynamical diffraction theory literature. Here, we define $\bar{\Lambda}_{H}$ in the same way as in [18]. With such a definition, the two characteristic measures of time in Bragg diffraction $\mathcal{T}_{d}(21)$ and $\mathcal{T}_{\Lambda}$ (42) have identical structure. In some other references, including [1,11,39], etc., the extinction length $\Lambda_{H}$ is defined to be a factor $2 \pi$ larger: $\Lambda_{H}=2 \pi \bar{\Lambda}_{H}$.

[27] Strictly speaking, the invariance in $\theta$ holds only for diffraction of linearly polarized fields in the $\sigma$-polarization state for which $P=1$. If the radiation field is $\pi$ polarized, then $P=\cos 2 \theta$, and $\bar{\Lambda}_{H}^{(\mathrm{s})}$ changes as $\left|\cos ^{-1} 2 \theta\right|$.

[28] S. Stoupin and Y. V. Shvyd'ko, Phys. Rev. Lett. 104, 085901 (2010).

[29] S. Stoupin and Y. V. Shvyd'ko, Phys. Rev. B 83, 104102 (2011).

[30] K. A. Gschneidner, Jr., in Solid State Physics, edited by F. Seitz and D. Turnbull (Academic Press, New York, 1964), Vol. 16, pp. 275-426.

[31] L. Kissel and R. Pratt, Acta Crystallogr. Sect. A 46, 170 (1990) [http://www-phys.llnl.gov/Research/scattering].

[32] L. Kissel, B. Zhou, S. Roy, S. K. S. Gupta, and R. H. Pratt, Acta Crystallogr. Sect. A 51, 271 (1995).

[33] Y. V. Shvyd'ko, S. Stoupin, A. Cunsolo, A. Said, and X. Huang, Nature Phys. 6, 196 (2010).

[34] Y. V. Shvyd'ko, S. Stoupin, V. Blank, and S. Terentyev, Nature Photon. 5, 539 (2011).

[35] J. Feldhaus, E. Saldin, J. Schneider, E. Schneidmiller, and M. Yurkov, Opt. Commun. 140, 341 (1997).

[36] E. L. Saldin, E. A. Schneidmiller, Y. V. Shvyd'ko, and M. V. Yurkov, Nucl. Instrum. Methods Phys. Res., Sect. A 475, 357 (2001).

[37] G. Geloni, V. Kocharyan, and E. Saldin, arXiv:1008.3036v1.

[38] G. Geloni, V. Kocharyan, and E. Saldin, J. Mod. Opt. 58, 1391 (2011)

[39] J. Amann, W. Berg, V. Blank, F.-J. Decker, Y. Ding, P. Emma, Y. Feng, J. Frisch, D. Fritz, J. Hastings et al., Nature Photon. 6, 693 (2012).

[40] A. Zholents, P. Heimann, M. Zolotorev, and J. Byrd, Nucl. Instrum. Methods Phys. Res., Sect. A 425, 385 (1999).

[41] Tables of Integral Transforms, edited by A. Erdelyi (McGraw-Hill Book Company, Inc., New York, 1954), Vol. I.

[42] I. S. Gradshteyn and I. M. Ryzhik, Table of Integrals, Series, and Products (Academic Press Inc., New York, 1965), p. 472, paragraph 3.876.

[43] M. v. Laue, Acta Crystallogr. 5, 619 (1952).

[44] N. Kato, Acta Crystallogr. 11, 885 (1958). 